\title{
Mean-field avalanche size exponent for sandpiles on Galton-Watson trees
}

\author{
Antal A. Járai ${ }^{1}$ - Wioletta M. Ruszel ${ }^{2,3}$ (D) Ellen Saada ${ }^{4}$ \\ Received: 4 July 2018 / Revised: 14 October 2019 / Published online: 3 November 2019 \\ (C) The Author(s) 2019
}

\begin{abstract}
We show that in Abelian sandpiles on infinite Galton-Watson trees, the probability that the total avalanche has more than $t$ topplings decays as $t^{-1 / 2}$. We prove both quenched and annealed bounds, under suitable moment conditions. Our proofs are based on an analysis of the conductance martingale of Morris (Probab Theory Relat Fields 125:259-265, 2003), that was previously used by Lyons et al. (Electron J Probab 13(58):1702-1725, 2008) to study uniform spanning forests on $\mathbb{Z}^{d}, d \geq 3$, and other transient graphs.
\end{abstract}

Keywords Abelian sandpile · Uniform spanning tree · Conductance martingale ·

Wired spanning forest

Mathematics Subject Classification $60 \mathrm{~K} 35 \cdot 82 \mathrm{C} 20$

\section{Introduction and results}

The Abelian sandpile model was introduced in 1988 by Bak, Tang and Wiesenfeld in [3] as a toy model displaying self-organized criticality. A self-organized critical

\footnotetext{
$凶 \quad$ Wioletta M. Ruszel

w.m.ruszel@uu.nl

Antal A. Járai

a.jarai@bath.ac.uk

Ellen Saada

ellen.saada@mi.parisdescartes.fr

1 Department of Mathematical Sciences, University of Bath, Claverton Down, Bath BA2 7AY, UK

2 Delft Institute of Applied Mathematics, Technische Universiteit Delft, Van Mourik Broekmanweg 6, 2628 XE Delft, The Netherlands

3 Present Address: Mathematical Institute, University of Utrecht, Budapestlaan 6, 3584 CD Utrecht, The Netherlands

4 CNRS, UMR 8145, Laboratoire MAP5, Université Paris Descartes, 45, rue des Saints Pères, 75270 Paris Cedex 06, France
} 
model is postulated to drive itself into a critical state which is characterized by powerlaw behaviour of, for example, correlation functions, without fine-tuning an external parameter. For a general overview we refer to [17,27] and to some of the physics literature $[8,9]$. There are connections of the sandpile model to Tutte polynomials [7], logarithmic conformal invariance [30], uniform spanning trees [8], and neuronal communication [4].

Consider a finite connected graph $G=(V \cup\{s\}, E)$ with a distinguished vertex $s$ called the sink. Assign to each vertex $x \in V$ a natural number $\eta_{x} \in \mathbb{N}$ representing its height or mass.

The Abelian sandpile model is defined as follows: choose at every discrete time step a vertex $x \in V$ uniformly at random and add mass 1 to it. If the resulting mass at $x$ is at least the number of neighbours of $x$, then we topple the vertex $x$ by sending unit mass to each neighbour of $x$. Mass can leave the system via the sink $s$, according to a rule depending on the graph. The topplings in $V$ will continue until all the vertices in $V$ are stable, that is, they have mass which is smaller than the number of neighbours. The sequence of consecutive topplings is called an avalanche. The order of topplings does not matter, hence the model is called Abelian. The unique stationary measure for this Markov chain is the uniform measure on the recurrent configurations.

There are various interesting quantities studied, for example the avalanche size or diameter distribution depending on the underlying graph $[5,10,14,16]$, the toppling durations, infinite-volume models [2,24], and continuous height analogues [19].

In particular, it is known that on a regular tree (Bethe lattice) the probability that an avalanche of size at least $t$ occurs, decays like a power law with mean-field exponent $-1 / 2$ for large $t$ [10], and the same is true on the complete graph [16]. Very recently, this has been extended by Hutchcroft [14] to a large class of graphs that are, in a suitable sense, high-dimensional. No assumptions of transitivity are needed in [14], but the proofs require bounded degree. In particular, [14] shows that the exponent $-1 / 2$ holds for the lattice $\mathbb{Z}^{d}$ for $d \geq 5$, and also for bounded degree non-amenable graphs. See also [5] for related upper and lower bounds on critical exponents on $\mathbb{Z}^{d}$ for $d \geq 2$.

In [28] sandpile models on random binomial (resp. binary) trees are considered, i.e. every vertex has two descendants with probability $p^{2}$, one with probability $2 p(1-p)$ and none with probability $(1-p)^{2}$ (resp. 2 offspring with probability $p$ and none with probability $1-p$ ); there, in a toppling, mass 3 is ejected by the toppling site, independently of its number of neighbours; hence there is dissipation (that is, there is mass which is not sent to a neighbouring site, but which is lost) when this number is less than 2. It is proven in [28] that in a small supercritical regime $p>1 / 2$ the (quenched and annealed) avalanche sizes decay exponentially, hence the model is not critical. Moreover (see [29]) the critical branching parameter for these models is $p=1$. The reason is that as soon as there exist vertices with degree strictly less than 2 , the extra dissipation thus introduced to the system is destroying the criticality of the model.

In this paper, we consider an Abelian sandpile model on a supercritical GaltonWatson branching tree $\mathbb{T}$ with possibly unbounded offspring distribution $\mathbf{p}=\left\{p_{k}\right\}_{k \geq 0}$ under some moment assumptions. We prove that the probability that the total avalanche has more than $t$ topplings decays as $t^{-1 / 2}$. Our proofs rely on a quantitative analysis 
of the conductance martingale of Morris [22,26], that he introduced to study uniform spanning forests on $\mathbb{Z}^{d}$ and other transient graphs). The use of this martingale is the major novelty of our paper, and our hope is that this gives insight into the behaviour of this martingale on more general graphs.

Our methods are very different from those of [14]. While the results of [14] are stated for bounded degree graphs (and more generally for networks with vertex conductances bounded away from 0 and infinity), Hutchcroft's approach can also be applied to unbounded degree graphs: In our context, under suitable moment conditions, the proof methods of [14] would yield the $t^{-1 / 2}$ behaviour with an extra power of $\log t$ present (T. Hutchcroft, personal communication).

We write $\nu_{\mathbb{T}}$ for the probability distribution of the sandpile model conditioned on the environment $\mathbb{T}$. Let $S$ denote the total number of topplings upon addition at the root, which is a.s. finite (see later on for details). Then we prove the following.

Theorem 1 Conditioned on the event that $\mathbb{T}$ survives, there exists $C=C(\mathbf{p})$ such that for all t large enough depending on $\mathbb{T}$ we have

$$
\nu_{\mathbb{T}}[S>t] \leq C t^{-1 / 2} .
$$

Furthermore if $\mathbf{p}$ has an exponential moment then there exists $c_{0}=c_{0}(\mathbb{T})$ that is a.s. positive on the event that $\mathbb{T}$ survives, such that we have

$$
v_{\mathbb{T}}[S>t] \geq c_{0} t^{-1 / 2} .
$$

We also have the following annealed bounds.

Theorem 2 Let $\mathbf{P}$ denote the probability distribution for the Galton-Watson trees, and $\mathbf{E}$ the corresponding expectation. There exists $C=C(\mathbf{p})>0$ such that

$$
\mathbf{E}\left[v_{\mathbb{T}}[S>t] \mid \mathbb{T} \text { survives }\right] \leq C t^{-1 / 2} .
$$

and if $\mathbf{p}$ has exponential moment then there exists $c=c(\mathbf{p})$ such that

$$
\mathbf{E}\left[\nu_{\mathbb{T}}[S>t] \mid \mathbb{T} \text { survives }\right] \geq c t^{-1 / 2}
$$

The paper is organized as follows. First in Sect. 2 we introduce the setting and notation and in particular we recall the decomposition of avalanches into waves. In Sect. 3 we prove upper bounds on the waves and in the subsequent Sect. 4 corresponding lower bounds. We deduce the corresponding bounds on $S$ from the bounds on the waves in Sect. 5 and finally we prove annealed bounds in Sect. 6.

\section{Notation and preliminaries}

\subsection{Abelian sandpile model on subtrees of the Galton-Watson tree}

We consider a supercritical Galton-Watson process with offspring distribution $\mathbf{p}=$ $\left\{p_{k}\right\}_{k \geq 0}$ with mean $\sum_{k \geq 0} k p_{k}>1$, starting with a single individual. 
Let us fix a realization $\mathbb{T}(\omega)$ of the family tree of this Galton-Watson process with root denoted by $o$. We will call

$$
F:=\{\mathbb{T} \text { survives }\}
$$

and assume that $\omega \in F$. The random environment $\mathbb{T}=\mathbb{T}(\omega)$ is defined on a probability space $(\Omega, \mathscr{G}, \mathbf{P})$. The edge set of $\mathbb{T}$ is denoted by $E(\mathbb{T})$. We use the notation $\mathbb{T}$ to refer to both the tree and to its vertex set. Take a subset $A \subset \mathbb{T}$ and let us denote by $\partial_{E} A$ the edge boundary of $A$, i.e. the set of edges $e=(v, u) \in E(\mathbb{T})$ such that $v \in A$ and $u \in A^{c}$, where $A^{c}$ is the complement of $A$ in $\mathbb{T}$. We denote by $|A|$ the cardinality of $A$. We say that $A$ is connected if the subgraph induced in $\mathbb{T}$ is connected. Then the distance $d(u, v)$ between the two vertices $u, v \in A$ is defined as the number of edges of the shortest path joining them within $A$. For $v \in \mathbb{T}$ we write $|v|=d(o, v)$. The (outer) vertex boundary $\partial_{V} A$ is defined as follows. A vertex $v \in \mathbb{T}$ belongs to $\partial_{V} A$ if $v \in A^{c}$ and there exists $u \in A$ such that $(u, v) \in E(\mathbb{T})$. Let $\partial_{V}^{\text {in }} A=\left\{v \in A: \exists w \in A^{c}\right.$ such that $\left.(v, w) \in E(\mathbb{T})\right\}$ be the internal vertex boundary of $A$. We will further use the notation $(V, o)$ for a graph with vertex set $V$ and root $o$.

By a result of Chen and Peres ([6, Corollary 1.3]) we know that conditioned on $F$ the tree $\mathbb{T}$ satisfies anchored isoperimetry, meaning that the edge boundary of a set containing a fixed vertex is larger than some positive constant times the volume. This isoperimetric inequality ensures an exponential growth condition on the random tree.

They proved (case (ii) in the proof of [6, Corollary 1.3]) that there exists $\delta_{0}=$ $\delta_{0}(\mathbf{p})>0$ and a random variable $N_{1}=N_{1}(\mathbb{T})$ that is a.s. finite on $F$, such that for any finite connected $o \in A \subset \mathbb{T}$ with $|A| \geq N_{1}$ we have

$$
\left|\partial_{E} A\right| \geq \delta_{0}|A|
$$

It also follows from the proof of [6, Corollary 1.3] that there exists $c_{1}=c_{1}(\mathbf{p})>0$ such that

$$
\mathbf{P}\left[N_{1} \geq n \mid F\right] \leq e^{-c_{1} n}, \quad n \geq 0 .
$$

We denote by $\mathbb{T}_{k}=\{v \in \mathbb{T}: d(o, v)=k\}$ (respectively $\mathbb{T}_{<k}=\{v \in \mathbb{T}: d(o, v)<$ $k\}$ ) the set of vertices at precisely distance $k$ (respectively at distance less than $k$ ) from the root, and analogously we define $\mathbb{T}_{\leq k}$. We write $\mathbb{T}(v)$ for the subtree of $\mathbb{T}$ rooted at $v$. For a vertex $v \in \mathbb{T}$ we denote by $\operatorname{deg}(v)$ the degree $\operatorname{deg}_{\mathbb{T}}(v)$ of vertex $v$ within $\mathbb{T}$ (i.e. the number of edges in $E(\mathbb{T})$ with one end equal to $v$ ), and we denote by $\operatorname{deg}^{+}(v)$ the forward degree $\operatorname{deg}_{\mathbb{T}}^{+}(v)$ of $v$, that is the number of children of $v$.

For some finite connected subset $H \subset \mathbb{T}$ such that $o \in H$ we write $\mathbb{T}_{H}^{*}$ for the finite connected wired graph, i.e. such that each vertex in $H^{c}$ is identified with some cemetery vertex $s$, called a sink. For a vertex $v \in H$ we denote by $\operatorname{deg}_{H}(v)$ the degree of vertex $v$ within $H$ (i.e. the number of edges in $E\left(\mathbb{T}_{H}^{*}\right)$ with one end equal to $v$ ), and we denote by $\operatorname{deg}_{H}^{+}(v)$ the forward degree of $v$ within $H$. We fix such a subset $H$ from now on.

We gather in the following subsections results we need on the Abelian sandpile model, for which we refer for instance to [8,12,17,27]. 


\subsubsection{Height configurations and legal topplings}

Height configurations on $\mathbb{T}_{H}^{*}$ are elements $\eta \in\{0,1,2, \cdots\}^{H}$. For $u \in H, \eta_{u}$ denotes the height at vertex $u$. A height configuration $\eta$ is stable if $\eta_{u} \in\left\{0,1,2, \ldots, \operatorname{deg}_{H}(u)-\right.$ 1 ) for all $u \in H$. Stable configurations are collected in the set $\Omega_{H}$. Note that $\operatorname{deg}_{H}(u)$, $u \in H$, and $\Omega_{H}$, depend on the realization of the Galton-Watson tree $\mathbb{T}$, hence are random.

For a configuration $\eta$, we define the toppling operator $T_{u}$ via

$$
\left(T_{u}(\eta)\right)_{v}=\eta_{v}-\Delta_{u v}^{H}
$$

where $\Delta^{H}$ is the toppling matrix, indexed by vertices $u, v \in H$ and defined by

$$
\Delta_{u v}^{H}= \begin{cases}\operatorname{deg}_{H}(u), & \text { if } u=v \\ -1, & \text { if }(u, v) \in E\left(\mathbb{T}_{H}^{*}\right) .\end{cases}
$$

In words, in a toppling at $u, \operatorname{deg}_{H}(u)$ particles are removed from $u$, and every neighbour of $u$ receives one particle. Note that $\Delta^{H}$ depends on the realization of $\mathbb{T}$ which hence is random in contrast to the case of the binary tree studied in [28]. Therefore there is no dissipation in a toppling, except for the particles received by the sink of $\mathbb{T}_{H}^{*}$.

A toppling at $u \in H$ in configuration $\eta$ is called legal if $\eta_{u} \geq \operatorname{deg}_{H}(u)$. A sequence of legal topplings is a composition $T_{u_{n}} \circ \cdots \circ T_{u_{1}}(\eta)$ such that for all $k=1, \cdots, n$ the toppling at $u_{k}$ is legal in $T_{u_{k-1}} \circ \cdots \circ T_{u_{1}}(\eta)$. The stabilization of a configuration $\eta$ is defined as the unique stable configuration $\mathrm{S}(\eta) \in \Omega_{H}$ that arises from $\eta$ by a sequence of legal topplings. Every $\eta \in\{0,1,2, \cdots\}^{H}$ can be stabilized thanks to the presence of a sink.

\subsubsection{Addition operator and Markovian dynamics}

Let $u \in H$, the addition operator is the map $a_{u}: \Omega_{H} \rightarrow \Omega_{H}$ defined via

$$
a_{u} \eta=\mathrm{S}\left(\eta+\delta_{u}\right)
$$

where $\delta_{u} \in\{0,1\}^{H}$ is such that $\delta_{u}(u)=1$ and $\delta_{u}(z)=0$ for $z \in H, z \neq u$. In other words, $a_{u} \eta$ is the effect of an addition of a single grain at $u$ in $\eta$, followed by stabilization.

The dynamics of the sandpile model can be defined as a discrete-time Markov chain $\{\eta(n), n \in \mathbb{N}\}$ on $\Omega_{H}$ with

$$
\eta(n)=\prod_{i=1}^{n} a_{X_{i}} \eta(0)
$$

where $X_{i}, 1 \leq i \leq n$, are i.i.d. uniformly chosen vertices in $H$. 


\subsubsection{Recurrent configurations, spanning trees and stationary measure}

The set of recurrent configurations $\mathrm{R}_{H}$ of the sandpile model corresponds to the recurrent states of the Markov chain (4) defined above. This Markov chain has a unique stationary probability measure $v_{H}$ which is the uniform measure on the set $\mathrm{R}_{H}$. There is a bijection between $\mathrm{R}_{H}$ and the spanning trees of $\mathbb{T}_{H}^{*}$ [25], that is useful in analyzing $v_{H}$.

Let $o \in H_{1} \subset H_{2} \subset \cdots \subset H_{n} \subset \cdots$ be a sequence of finite sets with union equal to $\mathbb{T}$. The sandpile measure $v_{\mathbb{T}}$ on $\mathbb{T}$ is defined as the weak limit of the stationary measures $v_{H_{n}}$ for the sandpile model on $\mathbb{T}_{H_{n}}^{*}$, when the limit exists. By [20, Theorem 3], an infinite volume sandpile measure $\nu_{\mathbb{T}}$ on $\mathbb{T}$ exists if each tree in the WSF (Wired Uniform Spanning Forest) on $\mathbb{T}$ has one end almost surely. The WSF is defined as the weak limit of the uniform spanning trees measure on $\mathbb{T}_{H_{n}}^{*}$, as $n \rightarrow \infty$. We refer to [23] for background on wired spanning forests. We define the related measure $\mathrm{WSF}_{o}$ in the following way. Identify $o$ and $s$ in $\mathbb{T}_{H_{n}}^{*}$ and let $\mathrm{WSF}_{o}$ be the weak limit of the uniform spanning tree in the resulting graph $G_{n}$ as $n \rightarrow \infty$. From now on, when working on a finite set $H$, we will abbreviate this procedure by $H \rightarrow \mathbb{T}$ (or $H$ goes to $\mathbb{T}$ ).

Let $\mathfrak{F}_{o}$ denote the connected component of $o$ under $\mathrm{WSF}_{o}$. Almost sure finiteness of $\mathfrak{F}_{o}$ is equivalent to one endedness of the component of $o$ under WSF, see [22]. The one end property for trees with bounded degree in the WSF of Galton-Watson trees was proven by [1, Theorem 7.2]. In the unbounded case it follows directly by [13, Theorem 2.1]. Draw a configuration from the measure $v_{\mathbb{T}}$, add a particle at $o$ and carry out all possible topplings. By [18, Theorem 3.11], one-endedness of the components and transience of $\mathbb{T}$ (for simple random walk) imply that there will be only finitely many topplings $\nu_{\mathbb{T}}$-a.s., and as a consequence the total number $S$ of topplings is a.s. finite.

\subsubsection{Waves, avalanches and Wilson's method}

Given a stable height configuration $\eta$ and $o \in H$, we define the avalanche cluster $\operatorname{Av}_{H}(\eta)$ induced by addition at $o$ in $\eta$ to be the set of vertices in $H$ that have to be toppled at least once in the course of the stabilization of $\eta+\delta_{o}$. Avalanches can be decomposed into waves (see $[15,18]$ ) corresponding to carrying out topplings in a special order. The first wave denotes the set of vertices in $H$ which have to be toppled in course of stabilization until $o$ has to be toppled again. The second wave starts again from $o$ and collects all the vertices involved in the toppling procedure until $o$ has to be toppled for the second time etc.

Let $N_{H}(\eta)$ denote the number of waves caused by addition at $o$ to the configuration $\eta$ in $H$. For fixed $\mathbb{T}$, the avalanche can be decomposed into

$$
\operatorname{Av}_{H}(\eta)=\bigcup_{i=1}^{N_{H}(\eta)} W_{H}^{i}(\eta)
$$

where $W_{H}^{i}(\eta)$ is the $i$-th wave. We write $W_{H}^{\text {last }}(\eta)$ for $W_{H}^{N_{H}(\eta)}(\eta)$. Further we denote by

$$
S_{H}(\eta)=\left|W_{H}^{1}(\eta)\right|+\cdots+\left|W_{H}^{\text {last }}(\eta)\right|
$$


the total number of topplings in the avalanche $\operatorname{Av}_{H}(\eta)$.

Note that waves can be defined on the full tree $\mathbb{T}$ as well where now it is possible to have infinitely many waves. However, due to the almost sure finiteness of the avalanche, $N_{H}$ under $v_{H}$ converges weakly to $N$ under the sandpile measure which is $v_{\mathbb{T}}$-a.s. finite. Furthermore $W_{H}^{i}$ converges weakly to $W^{i}$. We thus have

$$
\begin{aligned}
\operatorname{Av}(\eta) & =\bigcup_{i=1}^{N(\eta)} W^{i}(\eta) \\
S(\eta) & =\left|W^{1}(\eta)\right|+\cdots+\left|W^{\text {last }}(\eta)\right| \\
S(\eta) & \geq|\operatorname{Av}(\eta)| .
\end{aligned}
$$

Lemma 1 For any stable configuration $\eta$ on $\mathbb{T}$ we have the following.

(i) $W^{1}(\eta)$ equals the connected component of $o$ in $\left\{v \in \mathbb{T}: \eta_{v}=\operatorname{deg}(v)-1\right\}$ (possibly empty);

(ii) $N(\eta)=1+\max \left\{k \in \mathbb{N}: \mathbb{T}_{k} \subset W^{1}(\eta)\right\}$, with the right hand side interpreted as 0 when $W^{1}(\eta)=\emptyset$;

(iii) $W^{1}(\eta) \supset \cdots \supset W^{\text {last }}(\eta)$.

Proof (i) Call $A$ the connected component of $o$ in $\left\{v \in \mathbb{T}: \eta_{v}=\operatorname{deg}(v)-1\right\}$. Then all of the vertices in $A$ topple in the first wave (and they topple exactly once). On the other hand each vertex in $\partial_{V} A$ only receives one particle and hence will not topple.

(ii) After the first wave vertices other than $o$ in $\partial_{V}^{i n} W^{1}(\eta)$ have at most $\operatorname{deg}(v)-2$ particles and hence $W^{2}(\eta)$ equals the connected component of $o$ in $W^{1}(\eta) \backslash \partial_{V}^{i n} W^{1}(\eta)$. Let us call $K=\max \left\{k \in \mathbb{N}: \mathbb{T}_{k} \subset W^{1}(\eta)\right\}$. Then $\mathbb{T}_{\leq K} \subset W^{1}(\eta)$ but there exists $v \in \mathbb{T}_{K}$ such that $v \in \partial_{V}^{i n} W^{1}(\eta)$ and therefore $\mathbb{T}_{\leq K-1} \subset W^{2}(\eta)$ but $v \notin W^{2}(\eta)$. The claim follows now by repeating this argument for $\partial_{V}^{i n} W^{2}(\eta), W^{3}(\eta)$, etc. up to $W^{\text {last }}(\eta)$.

(iii) This last assertion follows from the arguments in the proof of (ii).

Recall that $\mathbb{T}$ is a fixed realization of a supercritical Galton-Watson tree. Observe that in the supercritical case, a.s. on $F$ there exists a vertex $v^{*}=v^{*}(\mathbb{T})$ such that $v^{*}$ has at least two children with an infinite line of descent, and $v^{*}$ is the closest such vertex to $o$. Hence, in the sequel we may assume without loss of generality that our sample $\mathbb{T}$ is such that $v^{*}$ exists.

Lemma 2 For $v_{\mathbb{T}}$-a.e. $\eta$ there is at most one wave with the property that $v^{*}$ topples but one of its children does not. When this happens, we have $N(\eta) \geq\left|v^{*}\right|+1$, and the wave in question is $W^{N-\left|v^{*}\right|}(\eta)$.

Proof Let $o=u_{0}, \ldots, u_{\left|v^{*}\right|}=v^{*}$ be the path from $o$ to $v^{*}$. Then for each $0 \leq k \leq$ $\left|v^{*}\right|-1$, the only child of $u_{k}$ with an infinite line of descent is $u_{k+1}$. This implies that the graph $H_{0}:=\mathbb{T} \backslash \mathbb{T}\left(v^{*}\right)$ is finite. Consider any finite subtree $H$ of $\mathbb{T}$ that contains $\left\{v^{*}\right\} \cup H_{0}$. By the burning test of Dhar $[8,12]$, under $v_{H}$ we have $\eta(w)=\operatorname{deg}(w)-1$ for all $w \in H_{0}$. Taking the weak limit, this also holds under $\nu_{\mathbb{T}}$ (which exists for a.e. $\mathbb{T}$ ). 
It follows from this and Lemma 1 that either $v^{*}$ does not topple in the avalanche (when $\eta\left(v^{*}\right) \leq \operatorname{deg}\left(v^{*}\right)-2$ ), or if $v^{*}$ topples, then there is an earliest wave $W^{\ell}(\eta)$ such that $v^{*}$ topples in $W^{\ell}(\eta)$, but one of its children does not. It follows then by induction that in $W^{\ell+k}(\eta)$ the vertex $u_{\left|v^{*}\right|-k}$ topples, but $u_{\left|v^{*}\right|-k+1}$ does not, for $1 \leq k \leq\left|v^{*}\right|$. Hence $\ell+\left|v^{*}\right|=N$, and the claim follows.

In addition to the above lemmas, we will use the following upper bound. Let $G^{\mathbb{T}}(x, y)=\left(\Delta^{\mathbb{T}}\right)^{-1}(x, y)$, where $\Delta^{\mathbb{T}}$ is the graph Laplacian of $\mathbb{T}$. This is the same as the Green's function of the continuous time simple random walk on $\mathbb{T}$ that crosses each edge at rate 1 .

Lemma 3 For $\eta$ sampled from $v_{\mathbb{T}}$ and the corresponding $W_{S} F_{o}$-measure we have

$$
\nu_{\mathbb{T}}\left(W^{1}(\eta) \in \mathscr{A}\right) \leq G^{\mathbb{T}}(o, o) W S F_{o}\left(\mathfrak{F}_{o} \in \mathscr{A}\right)
$$

where $\mathscr{A}$ is a cylinder event.

Proof We first show the statement in finite volume $H$ and then take the weak limit. Let $\overline{\mathrm{R}}_{H}$ be the set of configurations that appear just before a wave (thus each $\bar{\eta}$ satisfies $\left.\bar{\eta}(o)=\operatorname{deg}_{H}(o)\right)$, and write $W_{H}(\bar{\eta})$ for the set of vertices that topple in the wave represented by $\bar{\eta}$. By [15] there is a bijection between $\overline{\mathrm{R}}_{H}$ and 2-component spanning forest on $\mathbb{T}_{H}^{*}$ such that $o$ and $s$ are in different components. Alternatively these are spanning trees of the graph $G$ obtained from $\mathbb{T}_{H}^{*}$ by identifying $o$ and $s$. Let us call the uniform spanning tree measure on this finite graph $\mathrm{WSF}_{o, H}$. We have

$$
\begin{aligned}
v_{H}\left(W_{H}^{1}(\eta) \in \mathscr{A}\right) & =\frac{\left|\left\{\eta \in \mathrm{R}_{H}: W_{H}^{1}(\eta) \in \mathscr{A}\right\}\right|}{\left|\mathrm{R}_{H}\right|} \\
& \leq \frac{\left|\overline{\mathrm{R}}_{H}\right|}{\left|\mathrm{R}_{H}\right|} \cdot \frac{\left|\left\{\bar{\eta} \in \overline{\mathrm{R}}_{H}: W_{H}(\bar{\eta}) \in \mathscr{A}\right\}\right|}{\left|\overline{\mathrm{R}}_{H}\right|} \\
& =\mathbb{E}_{v_{H}}(N) \mathrm{WSF}_{o, H}\left(\mathfrak{F}_{o} \in \mathscr{A}\right)
\end{aligned}
$$

where the last step follows from the bijection. By Dhar's formula [8] and taking the weak limit $H \rightarrow \mathbb{T}$ (see Sect. 2.1.3) we conclude the claim.

Occasionally, we will use Wilson's algorithm [31], that provides a way to sample uniform spanning trees in finite graphs, and as such can be used to sample $\mathfrak{F}_{o}$ under $\mathrm{WSF}_{o, H}$, as follows. Enumerate $H \backslash\{o\}$ as $\left\{v_{1}, \ldots, v_{|H|-1}\right\}$. Run a loop-erased random walk (LERW) in $\mathbb{T}_{H}^{*}$ from $v_{1}$ until it hits $\{o, s\}$, which yields a path $\gamma_{1}$. Then run a LERW from $v_{2}$ until it hits $\gamma_{1} \cup\{o, s\}$, yielding a path $\gamma_{2}$, etc. The union of all the LERWs is a two component spanning forest with $o$ and $s$ in different components, and the component containing $o$ is distributed as $\mathfrak{F}_{o}$. By passing to the limit $H \rightarrow \mathbb{T}$ and using transience of $\mathbb{T}$, one obtains the following algorithm to sample $\mathfrak{F}_{o}$ under $\mathrm{WSF}_{o}$. Enumerate $\mathbb{T} \backslash\{o\}=\left\{v_{1}, v_{2}, \ldots\right\}$. Run a LERW from $v_{1}$, stopped if it hits $o$, yielding a path $\gamma_{1}$. Then run a LERW from $v_{2}$, stopped if it hits $\gamma_{1} \cup\{o\}$, yielding a path $\gamma_{2}$, etc.

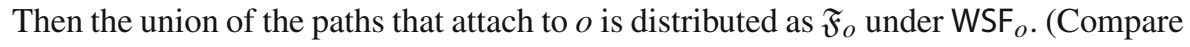
[23, Section 10.1] on Wilson's method rooted at infinity.) 


\subsection{Electrical networks and the conductance martingale}

\subsubsection{Effective conductances and resistances}

A general reference for this section is the book [23]. Let $G=(V, E)$ be a finite or locally finite infinite graph, for example $\mathbb{T}_{H}^{*}$ or $\mathbb{T}(v)$. We can regard them as an electrical network where each edge has conductance (and hence resistance) 1. An oriented edge $e=\left(e^{-}, e^{+}\right)$(or $e^{\rightarrow}$ ) has a head $e^{+}$and a tail $e^{-}$. The set of oriented edges is denoted by $E^{\rightarrow}$. In a finite network, the effective resistance $\mathscr{R}$ between two sets $A$ and $B$ will be denoted by $\mathscr{R}(A \leftrightarrow B)$. The effective conductance $\mathscr{C}$ between $A$ and $B$ is equal to

$$
\mathscr{C}(A \leftrightarrow B)=\frac{1}{\mathscr{R}(A \leftrightarrow B)} .
$$

In an infinite network $G$, we will need the effective resistance to infinity $\mathscr{R}(A \leftrightarrow$ $\infty ; G)$ and

$$
\mathscr{R}(A \leftrightarrow \infty ; G)=\frac{1}{\mathscr{C}(A \leftrightarrow \infty ; G)} .
$$

where $\mathscr{C}(A \leftrightarrow \infty ; G)$ denotes the effective conductance to infinity in $G$.

Since we are dealing with trees, we will often be able to compute resistances and conductances using series and parallel laws. If $G$ is a finite network and $\mathbf{T}$ is the uniform spanning tree of $G$ we can write

$$
\mathbb{P}(e \in \mathbf{T})=\mathscr{R}\left(e^{-} \leftrightarrow e^{+}\right)
$$

due to Kirchhoff's law [21]. For any vertex $v \in \mathbb{T}$ denote

$$
\mathscr{C}(v):=\mathscr{C}(v \leftrightarrow \infty ; \mathbb{T}(v)) \leq \operatorname{deg}^{+}(v),
$$

where the inequality follows since each edge has unit resistance.

The following lemma is a special case of a computation in the proof of the martingale property in [26, Theorem 6]. For convenience of the reader, we give here a short proof based on Wilson's algorithm, which is possible since we are dealing with trees.

Lemma 4 Let $o \in A \subset \mathbb{T}$ be connected, $B \subset \partial_{V} A$ and $e=\left(e^{-}, e^{+}\right) \in \partial_{E} A$ with $e^{+} \notin B$. Then we have

$$
W_{S F_{o}}\left(e^{+} \in \mathfrak{F}_{o} \mid A \subset \mathfrak{F}_{o}, B \cap \mathfrak{F}_{o}=\emptyset\right)=\frac{1}{1+\mathscr{C}\left(e^{+}\right)} .
$$

Proof Take $H$ large enough such that $A \cup B \cup\left\{e^{+}\right\} \subset H$ and let $G$ be the graph obtained from $\mathbb{T}_{H}^{*}$ by identifying $o$ and $s$. Let $\mathbb{T}_{H}^{*}\left(e^{+}\right)$be the subgraph of $\mathbb{T}_{H}^{*}$ induced by the vertices in $\left(\mathbb{T}\left(e^{+}\right) \cap H\right) \cup\{s\}$. Using Wilson's algorithm to sample $\mathrm{WSF}_{o, H}$, we have that $\mathrm{WSF}_{o, H}\left(e^{+} \in \mathfrak{F}_{o} \mid A \subset \mathfrak{F}_{o}, B \cap \mathfrak{F}_{o}=\emptyset\right)$ equals the probability that a simple 
random walk in $\mathbb{T}_{H}^{*}$ started at $e^{+}$hits $e^{-}$before hitting $s$. This equals $\left[1+\mathscr{C}\left(e^{+} \leftrightarrow\right.\right.$ $\left.\left.s ; \mathbb{T}_{H}^{*}\left(e^{+}\right)\right)\right]^{-1}$, and letting $H$ go to $\mathbb{T}$ we obtain the result.

\subsubsection{The conductance martingale}

Let us fix an environment $\mathbb{T}$, and let $\mathfrak{F}$ denote a sample from the measure $\mathrm{WSF}_{o}$ defined on the graph $\mathbb{T}$. Recall $\mathfrak{F}_{o}$ is the connected component of $o$ in $\mathfrak{F}$.

We inductively construct a random increasing sequence $E_{0} \subset E_{1} \subset E_{2} \subset \cdots$ of edges. Put $E_{0}=\emptyset$. Assuming $n \geq 0$ and that $E_{n}$ has been defined, let $S_{n}$ be the set of vertices in the connected component of $o$ in $E_{n} \cap \mathfrak{F}$ (we have $S_{0}=\{o\}$ ). Let us call all edges in $\mathbb{T} \backslash E_{n}$ that are incident to $S_{n}$ active at time $n$, and let us denote by $\mathscr{A}_{n}$ the event that this set of active edges is empty. On the event $\mathscr{A}_{n}$, that is, when all edges in $\mathbb{T}$ incident to $S_{n}$ belong to $E_{n}$, we set $E_{n+1}=E_{n}$. On the event $\mathscr{A}_{n}^{c}$, we select an active edge $e_{n+1}$, and we set $E_{n+1}=E_{n} \cup\left\{e_{n+1}\right\}$. (Note: at this point we have not yet specified how we select an active edge. In some cases this will not matter, in some other cases we will make a more specific choice later, see Sect. 3). Note that the event $\left\{\left|\mathfrak{F}_{o}\right|<\infty\right\}$ equals $\bigcup_{n \geq 1} \mathscr{A}_{n}$. Let

$$
M_{n}:=\mathscr{C}\left(S_{n} \leftrightarrow \infty ; \mathbb{T} \backslash E_{n}\right) .
$$

Let $\mathscr{F}_{n}$ denote the $\sigma$-field generated by $E_{n}$ and $E_{n} \cap \mathfrak{F}$. By a result of Morris (see [26, Theorem 8] and [22, Lemma 3.3]) $M_{n}$ is an $\mathscr{F}_{n}$-martingale.

Since we are dealing with trees, the increments of $M_{n}$ can be expressed very simply. Let $\mathscr{C}_{n}:=\mathscr{C}\left(e_{n+1}^{+}\right)(\mathrm{cf} .(7))$ and recall that this is the conductance from $e_{n+1}^{+}$to infinity in the subtree $\mathbb{T}\left(e_{n+1}^{+}\right)$. Then by Lemma 4 the probability, given $\mathscr{F}_{n}$, that $e_{n+1}$ belongs to $\mathfrak{F}_{o}$ equals $\left(1+\mathscr{C}_{n}\right)^{-1}$. On this event, we have

$$
M_{n+1}-M_{n}=-\frac{1}{1+\frac{1}{\mathscr{C}_{n}}}+\mathscr{C}_{n}=-\frac{\mathscr{C}_{n}}{1+\mathscr{C}_{n}}+\mathscr{C}_{n}=\frac{\mathscr{C}_{n}^{2}}{1+\mathscr{C}_{n}}
$$

Here the negative term is the conductance from $e_{n+1}^{-}$to infinity via the edge $e_{n+1}$. This implies that conditionally on $\mathscr{F}_{n}$ we have

$$
M_{n+1}-M_{n}= \begin{cases}\frac{\mathscr{C}_{n}^{2}}{1+\mathscr{C}_{n}} & \text { with probability } \frac{1}{1+\mathscr{C}_{n}} \\ -\frac{\mathscr{C}_{n}}{1+\mathscr{C}_{n}} & \text { with probability } \frac{\mathscr{C}_{n}}{1+\mathscr{C}_{n}}\end{cases}
$$

Let

$$
D_{i}=E^{\mathbb{T}}\left[M_{i+1}^{2}-M_{i}^{2} \mid \mathscr{F}_{i}\right]=\mathscr{C}_{i} \frac{\mathscr{C}_{i}^{2}}{\left(1+\mathscr{C}_{i}\right)^{2}}
$$

We will use the short notation $P^{\mathbb{T}}$ instead of $\mathrm{WSF}_{o}$ from now on and denote $E^{\mathbb{T}}$ the associated expectation. 


\section{Upper bound on waves}

In this section we give upper bounds on waves for general offspring distributions, conditioning the environment on the event $F$ (cf. (1)).

Let $\mathbb{T}^{\prime}$ denote the subtree of $\mathbb{T}$ consisting of those vertices $v$ such that $\mathbb{T}(v)$ is infinite. We will write

$$
\overline{\mathscr{C}}(v):=\max \{\mathscr{C}(v), 1\}
$$

Recall the random variable $N_{1}(\mathbb{T})$ from (2).

Theorem 3 Suppose that $1<\sum_{k \geq 0} k p_{k} \leq \infty$. There exist $C_{1}=C_{1}(\mathbf{p})$ and $t_{0}=t_{0}(\mathbf{p})$ such that on the event of survival we have

$$
P^{\mathbb{T}}\left[\left|\mathfrak{F}_{o}\right|>t\right] \leq C_{1} \mathscr{C}(o) t^{-1 / 2}, \quad t \geq \max \left\{t_{0}(\mathbf{p}), N_{1}(\mathbb{T})\right\}
$$

Therefore,

$$
P^{\mathbb{T}}\left[\left|\mathfrak{F}_{o}\right|>t\right] \leq C_{1} N_{1}^{1 / 2} \overline{\mathscr{C}}(o) t^{-1 / 2}, \quad t>0
$$

We will use the following stopping times:

$$
\begin{aligned}
\tau^{-} & =\inf \left\{n \geq 0: M_{n}=0\right\} \\
\tau_{b, t} & =\inf \left\{n \geq 0: M_{n} \geq b t^{1 / 2}\right\}, \quad b>0, t>0 .
\end{aligned}
$$

We impose the following restriction on selecting edges to examine for the martingale. If there is an active edge $e$ available with $\mathscr{C}\left(e^{+}\right)^{2} /\left(1+\mathscr{C}\left(e^{+}\right)\right)<(1 / 2) t^{1 / 2}$, we select one such edge to examine, otherwise we select any other edge.

Observe that on the event $F$, we have $M_{0}>0$ (recall that $M_{0}=\mathscr{C}(o)$ ), and Doob's inequality gives

$$
P^{\mathbb{T}}\left[\tau_{1 / 4, t}<\tau^{-}\right] \leq P^{\mathbb{T}}\left[\sup _{n} M_{n} \geq \frac{1}{4} t^{1 / 2}\right] \leq 4 M_{0} t^{-1 / 2} .
$$

Moreover, as long as $n<\tau^{-}$, we have $M_{n}>0$. Consider the stopping time

$$
\sigma=\tau_{1 / 4, t} \wedge \inf \left\{n \geq 0: \frac{\mathscr{C}\left(e^{+}\right)^{2}}{1+\mathscr{C}\left(e^{+}\right)} \geq \frac{1}{2} t^{1 / 2} \text { for all active } e \text { at time } n\right\} \text {. }
$$

When there are no active edges at all, that is, at time $\tau^{-}$, the condition on them holds vacuously, and hence $\sigma \leq \tau^{-} \wedge \tau_{1 / 4, t}$.

Lemma 5 On the event $\left\{\sigma<\tau^{-}\right\}$,

(i) we have $M_{\sigma} \leq t^{1 / 2}$;

(ii) we either have the event $\left\{\tau_{1 / 4, t}<\tau^{-}\right\}$or else no edges are added to the cluster after time $\sigma$, that is: $\mathfrak{F}_{\sigma}=\mathfrak{F}_{n}=\mathfrak{F}_{\tau^{-}}$for all $\sigma \leq n \leq \tau^{-}$. 
Proof (i) The claim amounts to showing that when $M_{\sigma} \geq \frac{1}{4} t^{1 / 2}$, we have $M_{\sigma} \leq t^{1 / 2}$ (if $M_{\sigma} \leq \frac{1}{4} t^{1 / 2}$, then $M_{\sigma} \leq t^{1 / 2}$ ). Let $e$ be the edge examined at time $\sigma-1$. Then

$$
M_{\sigma} \leq M_{\sigma-1}+\frac{\mathscr{C}\left(e^{+}\right)^{2}}{1+\mathscr{C}\left(e^{+}\right)} \leq \frac{1}{4} t^{1 / 2}+\frac{1}{2} t^{1 / 2}<t^{1 / 2} .
$$

(ii) Let us assume that $M_{\sigma}<\frac{1}{4} t^{1 / 2}$ (otherwise the event $\left\{\tau_{1 / 4, t}<\tau^{-}\right\}$has occurred). Let $e_{1}, \ldots, e_{\ell}$ be the available edges at time $\sigma$. Examine each of the edges $e_{1}, \ldots, e_{\ell}$ in turn, to determine whether they belong to $\mathfrak{F}_{o}$ or not. Suppose that for some $1 \leq j \leq \ell$ we have that $e_{j}$ is found to belong to $\mathfrak{F}_{o}$, and let $j$ be the minimal such index. Then (recall the definition of $\sigma$ )

$$
M_{\sigma+j}=M_{\sigma+j-1}+\frac{\mathscr{C}\left(e_{j}^{+}\right)^{2}}{1+\mathscr{C}\left(e_{j}^{+}\right)}>\frac{\mathscr{C}\left(e_{j}^{+}\right)^{2}}{1+\mathscr{C}\left(e_{j}^{+}\right)} \geq \frac{1}{2} t^{1 / 2}>\frac{1}{4} t^{1 / 2} .
$$

Thus the event $\left\{\tau_{1 / 4, t}<\tau^{-}\right\}$occurs. This proves our claim.

We have

$$
M_{0}^{2}=E^{\mathbb{T}}\left[M_{\sigma}^{2} \mathbf{1}_{\sigma<\tau^{-}}\right]-E^{\mathbb{T}}\left[\sum_{i=0}^{\sigma-1} D_{i}\right] .
$$

Here, due to Lemma 5(i), the first term is bounded above by

$$
E^{\mathbb{T}}\left[M_{\sigma}^{2} \mathbf{1}_{\sigma<\tau^{-}}\right] \leq t^{1 / 2} E^{\mathbb{T}}\left[M_{\sigma} \mathbf{1}_{\sigma<\tau^{-}}\right]=t^{1 / 2} M_{0},
$$

and hence

$$
E^{\mathbb{T}}\left[\sum_{i=0}^{\sigma-1} D_{i}\right] \leq M_{0} t^{1 / 2}
$$

The idea is to show that there cannot be many active edges at time $\sigma$ from which the conductance is low, and hence there are sufficiently many terms $D_{i}$ such that $D_{i}>c$ for some $c>0$.

Recall the anchored isoperimetry equation (2) and exponential bound (3). The following proposition gives a bound on the probability of there being any connected subset of the Galton-Watson tree that has 'many' boundary edges with low conductance to infinity. Let $o \in A \subset \mathbb{T}$ be a finite connected set of vertices such that $|A|=n$. Let us call $e \in \partial_{E} A \delta$-good if $\mathscr{C}\left(e^{+}\right) /\left(1+\mathscr{C}\left(e^{+}\right)\right) \geq \delta$. Let us say that $A$ is $\delta$-good if

$$
\mid\left\{e \in \partial_{E} A: e \text { is } \delta \text {-good }\right\}|\geq \delta| \partial_{E} A \mid .
$$

We are going to need the isoperimetric profile function (see [23, Section 6.8]) given by:

$$
\psi(A, t):=\inf \left\{\left|\partial_{E} K\right|: A \subset K, K / A \text { connected, } t \leq|K|_{\text {deg }}<\infty\right\}
$$

where $|K|_{\text {deg }}=\sum_{v \in K} \operatorname{deg}(v)$. 
Proposition 1 Assume $1<\sum_{k \geq 0} k p_{k} \leq \infty$. There exists $\delta_{1}=\delta_{1}(\mathbf{p})>0$ such that all finite connected sets $A$ with $o \in A \subset \mathbb{T}$ and $|A| \geq N_{1}$ are $\delta_{1 \text {-good. }}$

Proof Observe that if $o \in A$ and $A$ is connected, then any $K$ inside the infimum in (11) is a tree, and hence

$$
|K|_{\operatorname{deg}}=\sum_{v \in K} \operatorname{deg}(v)=2|K|-2+\left|\partial_{E} K\right|
$$

This implies that if $|A| \geq N_{1}(\mathbb{T})$, we have

$$
\frac{\left|\partial_{E} K\right|}{|K|_{\text {deg }}}=\frac{\left|\partial_{E} K\right|}{2|K|-2+\left|\partial_{E} K\right|} \geq \frac{\left|\partial_{E} K\right|}{2|K|+\left|\partial_{E} K\right|} \geq \frac{\delta_{0}|K|}{2|K|+\delta_{0}|K|}=\frac{\delta_{0}}{2+\delta_{0}} .
$$

Consequently,

$$
\psi(A, t) \geq \frac{\delta_{0}}{2+\delta_{0}} t=: f(t)
$$

Therefore, an application of [23, Theorem 6.41] (which gives an upper bound of the effective resistance in terms of integrals over the lower bound of the isoperimetric profile function) yields that

$$
\mathscr{R}(A \leftrightarrow \infty) \leq \int_{|A|_{\operatorname{deg}}}^{\infty} \frac{16}{f(t)^{2}} d t=\frac{16\left(2+\delta_{0}\right)^{2}}{\delta_{0}^{2}}|A|_{\mathrm{deg}}^{-1} .
$$

Hence

$$
\mathscr{C}(A \leftrightarrow \infty) \geq \frac{\delta_{0}^{2}}{16\left(2+\delta_{0}\right)^{2}}|A|_{\mathrm{deg}} \geq \frac{\delta_{0}^{2}}{16\left(2+\delta_{0}\right)^{2}}\left|\partial_{E} A\right|
$$

Put

$$
\delta_{1}=\frac{1}{2}\left(\frac{\delta_{0}^{2}}{16\left(2+\delta_{0}\right)^{2}}\right)
$$

Since

$$
\mathscr{C}(A \leftrightarrow \infty)=\sum_{e \in \partial_{E} A} \frac{\mathscr{C}\left(e^{+}\right)}{1+\mathscr{C}\left(e^{+}\right)},
$$

we have that

$$
\left|\left\{e \in \partial_{E} A: \frac{\mathscr{C}\left(e^{+}\right)}{1+\mathscr{C}\left(e^{+}\right)} \geq \delta_{1}\right\}\right| \geq \delta_{1}\left|\partial_{E} A\right|,
$$

which is the claimed inequality. 
Proof of Theorem 3 Recall the positive constant $\delta_{0}$ from (2), the positive constant $\delta_{1}$ of Proposition 1, and the a.s. finite random variable $N_{1}=N_{1}(\mathbb{T})$ of (3).

Assume that $\mathbb{T}$ satisfies the event $\left\{N_{1}(\mathbb{T}) \leq t\right\}$. On the event

$$
\left\{\sup _{n} M_{n} \leq \frac{1}{4} t^{1 / 2}\right\} \cap\left\{\mid\left(\text { edges in } \mathfrak{F}_{o}\right) \mid>t-1\right\}
$$

we have $\left|\mathfrak{F}_{o}\right| \geq N_{1}$. Hence by the anchored isoperimetry equation (2) and by Proposition 1 we have

$$
\begin{aligned}
& \mid\left(\text { edges in } \mathfrak{F}_{o}\right)|=| \mathfrak{F}_{o} \mid-1 \\
& \quad \leq \frac{1}{\delta_{0}}\left|\partial_{E} \mathfrak{F}_{o}\right| \\
& \quad \leq \frac{1}{\delta_{0} \delta_{1}} \mid\left(\text { edges } e \text { in } \partial_{E} \mathfrak{F}_{o} \text { with } \frac{\mathscr{C}\left(e^{+}\right)}{1+\mathscr{C}\left(e^{+}\right)} \geq \delta_{1}\right) \mid \\
& \quad \leq \frac{1}{\delta_{0} \delta_{1}}\left(\frac{1}{\delta_{1}^{3}} \sum_{i=0}^{\sigma-1} D_{i}+\mid\left(\text { edges in } \partial_{E} \mathfrak{F}_{o} \text { examined after time } \sigma-1\right) \mid\right)
\end{aligned}
$$

where the last inequality used that when $\mathscr{C}_{i} /\left(1+\mathscr{C}_{i}\right) \geq \delta_{1}$, we have (recall (8))

$$
D_{i}=\mathscr{C}_{i} \frac{\mathscr{C}_{i}^{2}}{\left(1+\mathscr{C}_{i}\right)^{2}} \geq \delta_{1}^{3}
$$

In order to estimate the last term in the right hand side of (12), we use that if $e_{1}, \ldots, e_{\ell}$ are the edges that are examined after time $\sigma$, then on the event $\left\{\sup _{n} M_{n}<(1 / 4) t^{1 / 2}\right\}$, we have

$$
(1 / 4) t^{1 / 2}>M_{\sigma}=\sum_{j=1}^{\ell} \frac{\mathscr{C}\left(e_{j}^{+}\right)}{1+\mathscr{C}\left(e_{j}^{+}\right)} \geq \ell \frac{(1 / 2) t^{1 / 2}}{1+(1 / 2) t^{1 / 2}}=\ell \frac{1}{1+2 t^{-1 / 2}} \geq \ell\left(1-2 t^{-1 / 2}\right)
$$

and hence for $t \geq 16$ we have

$$
\ell \leq \frac{(1 / 4) t^{1 / 2}}{1-2 t^{-1 / 2}} \leq(1 / 2) t^{1 / 2}
$$

This gives that the right hand side of (12) is at most

$$
\frac{1}{\delta_{0} \delta_{1}^{4}} \sum_{i=0}^{\sigma-1} D_{i}+\frac{1}{\delta_{0} \delta_{1}} \frac{t^{1 / 2}}{2}
$$


The inequality (10) implies that

$$
P^{\mathbb{T}}\left[\sum_{i=0}^{\sigma-1} D_{i}>\frac{t \delta_{0} \delta_{1}^{4}}{2}\right] \leq \frac{2 M_{0}}{\delta_{0} \delta_{1}^{4}} t^{-1 / 2}
$$

Therefore, if $t \geq t_{0}:=\left(\delta_{0} \delta_{1}\right)^{-2}$ and $\mathbb{T}$ satisfies the event $\left\{N_{1}(\mathbb{T}) \leq t\right\}$, we have $\frac{1}{\delta_{0} \delta_{1}} \frac{t^{1 / 2}}{2} \leq \frac{t}{2}$, and hence for all $t \geq t_{0}$ we have

$$
\begin{aligned}
P^{\mathbb{T}} & {\left[\left|\mathfrak{F}_{o}\right|>t\right]=P^{\mathbb{T}}\left[\#\left(\text { edges in } \mathfrak{F}_{o}\right)>t-1\right] } \\
& \leq P^{\mathbb{T}}\left[\sup _{n} M_{n} \geq \frac{1}{4} t^{1 / 2}\right]+P^{\mathbb{T}}\left[\sup _{n} M_{n}<\frac{1}{4} t^{1 / 2}, \frac{1}{\delta_{0} \delta_{1}^{4}} \sum_{i=0}^{\sigma-1} D_{i}>\frac{t}{2}\right] \\
& \leq 4 M_{0} t^{-1 / 2}+\frac{2 M_{0}}{\delta_{0} \delta_{1}^{4}} t^{-1 / 2} \\
& =\mathscr{C}(o)\left[4+\frac{2}{\delta_{0} \delta_{1}^{4}}\right] t^{-1 / 2} .
\end{aligned}
$$

This completes the proof of the first statement, for $t \geq \max \left\{t_{0}(\mathbf{p}), N_{1}(\mathbb{T})\right\}$. The second statement of the theorem follows immediately, since $C_{1}>1$, and also $N_{1}^{1 / 2} t^{-1 / 2}>1$ if $t<N_{1}$.

\section{Lower bound on waves}

In this section we prove the lower bound corresponding to Theorem 3 . Denote by $f$ the generating function of $\mathbf{p}$, that is $f(z)=\sum_{k \geq 0} p_{k} z^{k}$. We introduce the following assumption on $f$ :

$$
\text { there exists } z_{0}:=e^{\beta_{0}}>1 \text { such that } f\left(z_{0}\right)<\infty \text {. }
$$

Theorem 4 Suppose that $\mathbf{p}$ satisfies Assumption $(\mathrm{M}-\beta)$ with some $\beta_{0}>0$, and suppose that $\sum_{k \geq 0} k p_{k}>1$. Then conditioned on $F$ there exists $c=c(\mathbb{T})>0$ such that

$$
P^{\mathbb{T}}\left[\left|\mathfrak{F}_{o}\right|>t\right] \geq c t^{-1 / 2}
$$

We will need the following a.s. upper bound on the vertex boundary of sets.

Proposition 2 Under Assumption $(\mathrm{M}-\beta)$, there exists an a.s. finite $C^{\prime}=C^{\prime}(\mathbb{T})$, such that for any finite connected set $o \in A \subset \mathbb{T}$ we have

$$
\left|A \cup \partial_{V} A\right| \leq C^{\prime}|A|
$$


Proof Fix a plane tree $A$ (i.e. $A$ is a rooted tree with root $o$ and the children of each vertex of $A$ are ordered). Also fix numbers $n_{v}, m_{v}$ for $v \in A$, with the following properties:

$$
\begin{aligned}
n_{v} & =\text { number of children of } v \text { in } A \\
n & :=|A|=\sum_{v \in A} n_{v}+1 \\
m_{v} & \geq 0 \\
d_{v} & :=n_{v}+m_{v} \\
M & :=\sum_{v \in A} m_{v} .
\end{aligned}
$$

For each $v \in A$, fix a subset $I_{v} \subset\left\{1, \ldots, d_{v}\right\}$ such that $\left|I_{v}\right|=n_{v}$. If we view $A$ as a subtree of $\mathbb{T}$ then every vertex $v \in A$ has forward degree $n_{v}$ in $A$ and forward degree $d_{v}$ in $\mathbb{T}$. Thus each $v \in A$ has $m_{v}$ children in $\mathbb{T}$ which belong to $\partial_{V} A$. We define the event

$E\left(A,\left\{m_{v}\right\},\left\{I_{v}\right\}\right)=\left\{\begin{array}{l}(\mathbb{T}, o) \text { has a rooted subtree }\left(A^{\prime}, o\right) \text { isomorphic to } \\ (A, o) \text { such that the forward degree in } \mathbb{T} \text { of each } v \in A^{\prime} \text { equals } d_{v} \\ \text { and the set of children in } A^{\prime} \text { of each } v \in A^{\prime} \text { equals } I_{v}\end{array}\right\}$

The probability of $E\left(A,\left\{m_{v}\right\},\left\{I_{v}\right\}\right)$ equals

$$
\mathbf{P}\left[E\left(A,\left\{m_{v}\right\},\left\{I_{v}\right\}\right)\right]=\prod_{v \in A} p\left(d_{v}\right)=\prod_{v \in A} p\left(n_{v}+m_{v}\right)
$$

where for readability we wrote $p\left(d_{v}\right)$ and $p\left(n_{v}+m_{v}\right)$ instead of $p_{d_{v}}$ and $p_{n_{v}+m_{v}}$. Hence, if $1<e^{\beta}<z_{0}$, we have

$$
\mathbf{P}\left[E\left(A,\left\{m_{v}\right\},\left\{I_{v}\right\}\right)\right]=\exp (-\beta M) \prod_{v \in A} p\left(n_{v}+m_{v}\right) e^{\beta m_{v}} .
$$

Let

$$
E^{\prime}\left(A,\left\{m_{v}\right\}\right)=\left\{\begin{array}{c}
(\mathbb{T}, o) \text { has a rooted subtree }\left(A^{\prime}, o\right) \text { isomorphic to }(A, o) \\
\text { such that the forward degree in } \mathbb{T} \text { of each } v \in A^{\prime} \text { equals } d_{v}
\end{array}\right\} .
$$

Taking a union bound in (14) and summing over $\left\{I_{v}\right\}$ yields:

$$
\begin{aligned}
& \mathbf{P}\left[E^{\prime}\left(A,\left\{m_{v}\right\}\right)\right] \\
& \quad \leq \exp (-\beta M) \prod_{v \in A}\left(\begin{array}{c}
n_{v}+m_{v} \\
n_{v}
\end{array}\right) p\left(n_{v}+m_{v}\right) e^{\beta m_{v}} \\
& \quad=\exp (-\beta M) \prod_{v \in A} \frac{1}{n_{v} !}\left(m_{v}+n_{v}\right) \cdots\left(m_{v}+1\right) p\left(n_{v}+m_{v}\right) e^{\beta m_{v}} .
\end{aligned}
$$


In order to sum over $m_{v}$, we are going to use that

$$
\sum_{m \geq 0}\left(\begin{array}{c}
n+m \\
n
\end{array}\right) p(n+m) z^{m}=\frac{1}{n !} \sum_{m \geq 0} p(n+m)(m+n) \cdots(m+1) z^{m}=\frac{1}{n !} f^{(n)}(z) .
$$

For a fixed $\tilde{M}$, let us define

$$
E^{\prime \prime}(A, \widetilde{M})=\left\{\begin{array}{l}
(\mathbb{T}, o) \text { has a rooted subtree }\left(A^{\prime}, o\right) \text { isomorphic to } \\
(A, o) \text { such that }\left|\partial_{V} A^{\prime}\right| \geq \widetilde{M}
\end{array}\right\} .
$$

Recall that $1<z_{1}:=e^{\beta}<z_{0}$. Fix some $C^{\prime \prime}$ and sum (15) over all $\left\{m_{v}\right\}$, with $M \geq \widetilde{M}:=\left(C^{\prime \prime}-1\right) n$. This gives

$$
\mathbf{P}\left[E^{\prime \prime}(A, \tilde{M})\right] \leq \exp \left(-\beta\left(C^{\prime \prime}-1\right) n\right) \prod_{v \in A} \frac{1}{n_{v} !} f^{\left(n_{v}\right)}\left(z_{1}\right)
$$

Due to Cauchy's theorem, we have

$$
\frac{1}{n_{v} !} f^{\left(n_{v}\right)}\left(z_{1}\right) \leq f\left(z_{0}\right) \frac{1}{\left(z_{0}-z_{1}\right)^{n_{v}+1}} \leq f\left(z_{0}\right) C^{n_{v}+1} .
$$

Substituting this into (16) and summing over $A$, while keeping $n$ fixed, yields

$$
\begin{aligned}
& \mathbf{P}\left[\exists \text { connected set } o \in A \subset \mathbb{T} \text { with }|A|=n \text { such that }\left|\partial_{V} A\right|>\left(C^{\prime \prime}-1\right) n\right] \\
& \quad \leq \exp \left(-\beta\left(C^{\prime \prime}-1\right) n\right) 4^{n} f\left(z_{0}\right)^{n} C^{2 n-1} .
\end{aligned}
$$

Here we used that there are $\leq 4^{n}$ non-isomorphic rooted plane trees $(A, o)$ of $n$ vertices. (This can be seen by considering the depth-first search path of $A$ starting from $o$, which gives an encoding of the tree by a simple random walk path of length $2 n$.) If $C^{\prime \prime}$ is sufficiently large, the estimate in the right hand side is summable in $n \geq 1$, and hence we have $\left|A \cup \partial_{V} A\right| \leq C^{\prime \prime}|A|=C^{\prime \prime} n$ for all but finitely many $n$. Increasing $C^{\prime \prime}$ to some $C^{\prime}$ if necessary, yields the claim (13) on the size of the boundary.

Lemma 6 Under Assumption (M- $\beta$ ), there exists an a.s. finite $C=C(\mathbb{T})$ such that

$$
E^{\mathbb{T}}\left[\tau^{-} \wedge t\right] \leq C t^{1 / 2}, \quad t \geq 1
$$

Proof Note that the set of edges examined by the conductance martingale up to time $\tau^{-}$equals the edges in $\mathfrak{F}_{o}$ union the edge boundary of $\mathfrak{F}_{o}$. Thus $\tau^{-}=\left|\mathfrak{F}_{o}\right|-1+\left|\partial_{V} \mathfrak{F}_{o}\right|$. Using (13) of Proposition 2, we have

$$
P^{\mathbb{T}}\left[\tau^{-} \geq s\right] \leq P^{\mathbb{T}}\left[\left|\mathfrak{F}_{o} \cup \partial_{V} \mathfrak{F}_{o}\right| \geq s\right] \leq P^{\mathbb{T}}\left[\left|\mathfrak{F}_{o}\right| \geq\left(1 / C^{\prime}\right) s\right]
$$

The right hand side is at most $C s^{-1 / 2}$, due to Theorem 3 . Summing over $1 \leq s \leq t$ proves the claim. 
We need one more proposition for the proof of Theorem 4.

Proposition 3 Under Assumption $(\mathrm{M}-\beta)$, there exists an a.s. finite $C=C(\mathbb{T})$ such that

$$
\sum_{i=0}^{\tau^{-} \wedge t-1} D_{i} \leq C\left(\tau^{-} \wedge t\right)
$$

Proof Let $A$ be the connected subgraph of $\mathbb{T}$ consisting of the edges inside $\mathfrak{F}_{o}$ that have been examined by time $\tau^{-} \wedge t$ and found to be in $\mathfrak{F}_{o}$. Then $|A| \leq \tau^{-} \wedge t$. For times $i$ such that the edge $e_{i}=\left(e_{i}^{-}, e_{i}^{+}\right)$examined at time $i$ was found to be in $\mathfrak{F}_{o}$, we use the bound (cf. (7), (8))

$$
D_{i}=\mathscr{C}_{i} \frac{\mathscr{C}_{i}^{2}}{\left(1+\mathscr{C}_{i}\right)^{2}} \leq \mathscr{C}_{i} \leq \operatorname{deg}^{+}\left(e_{i}^{+}\right)
$$

The sum of $D_{i}$ over such $i$ is hence bounded by $\left|A \cup \partial_{V} A\right|$. We can bound the sum of $D_{i}$ over the rest of the times by $\left|\partial_{V}\left(A \cup \partial_{V} A\right)\right|$. Due to Proposition 2, there exists an a.s. finite $C=C(\mathbb{T})$ such that

$$
\sum_{i=0}^{\tau^{-} \wedge t-1} D_{i} \leq\left|A \cup \partial_{V} A\right|+\sum_{w \in \partial_{V} A} \mathscr{C}(w) \leq C^{\prime}|A|+\left(C^{\prime}\right)^{2}|A| \leq C\left(\tau^{-} \wedge t\right) .
$$

Proof of Theorem 4 Recall that on the event $F=\{\mathbb{T}$ survives $\}$ we have that $M_{0}>0$. Using Proposition 3 and Lemma 6, we write

$$
\begin{aligned}
E^{\mathbb{T}}\left[M_{t}^{2}\right] & =E^{\mathbb{T}}\left[M_{t}^{2} \mathbf{1}_{\tau^{-}>t}\right]=M_{0}^{2}+E^{\mathbb{T}}\left[\sum_{i=0}^{\tau^{-} \wedge t-1} D_{i}\right] \leq M_{0}^{2}+C E^{\mathbb{T}}\left[\tau^{-} \wedge t\right] \\
& \leq M_{0}^{2}+C t^{1 / 2} \leq C^{\prime \prime} t^{1 / 2} .
\end{aligned}
$$

This gives

$$
M_{0}=E^{\mathbb{T}}\left[M_{t}\right]=E^{\mathbb{T}}\left[M_{t} \mathbf{1}_{\tau^{-}>t}\right] \leq\left(E^{\mathbb{T}}\left[M_{t}^{2}\right]\right)^{1 / 2} P^{\mathbb{T}}\left[\tau^{-}>t\right]^{1 / 2},
$$

and hence

$$
P^{\mathbb{T}}\left[\tau^{-}>t\right] \geq \frac{M_{0}^{2}}{C^{\prime \prime} t^{1 / 2}}
$$

This gives, using (13) of Proposition 2, that

$$
\begin{aligned}
P^{\mathbb{T}}\left[\left|\mathfrak{F}_{o}\right| \geq t\right] & \geq P^{\mathbb{T}}\left[\left|\mathfrak{F}_{o} \cup \partial_{V} \mathfrak{F}_{o}\right| \geq C^{\prime} t\right] \\
& =P^{\mathbb{T}}\left[\left|\mathfrak{F}_{o}\right|-1+\left|\partial_{V} \mathfrak{F}_{o}\right| \geq C^{\prime} t-1\right]
\end{aligned}
$$




$$
\begin{aligned}
& =P^{\mathbb{T}}\left[\tau^{-} \geq C^{\prime} t-1\right] \\
& \geq c_{4} t^{-1 / 2} .
\end{aligned}
$$

\section{From waves to avalanches}

We use the following decomposition of the supercritical branching process (see [23, Section 5.7]). Recall the definition of the subtree $\mathbb{T}^{\prime}$ of $\mathbb{T}$ : for any $v \in \mathbb{T}$ such that $\mathbb{T}(v)$ is finite, we remove all vertices of $\mathbb{T}(v)$ from $\mathbb{T}$, and hence $\mathbb{T}^{\prime}$ consists of those vertices of $\mathbb{T}$ with an infinite line of descent. Note that $o \in \mathbb{T}^{\prime}$. Let $\left\{\tilde{p}_{k}\right\}_{k \geq 0}$ be the offspring distribution of $\mathbb{T}$ conditioned on extinction. Then $\mathbb{T}$ can be obtained from $\mathbb{T}^{\prime}$ as follows. Let $\left\{\widetilde{\mathbb{T}}^{v}: v \in \mathbb{T}^{\prime}\right\}$ be i.i.d. family trees with offspring distribution $\left\{\widetilde{p}_{k}\right\}_{k \geq 0}$. Identify the root of $\widetilde{T}^{v}$ with vertex $v$ of $\mathbb{T}^{\prime}$. Then

$$
\mathbb{T}^{\prime} \cup\left(\cup_{v \in \mathbb{T}^{\prime}} \widetilde{\mathbb{T}}^{v}\right) \stackrel{\text { dist }}{=} \mathbb{T}
$$

Lemma 7 Let $v \in \mathbb{T}^{\prime}$. On the event $v \in \mathfrak{F}_{o}$, we also have $\widetilde{\mathbb{T}}^{v} \subset \mathfrak{F}_{o}$.

Proof Use Wilson's algorithm to generate $\mathfrak{F}_{o}$ by first starting a random walk at $v$. If this walk hits $o$, all vertices of $\widetilde{\mathbb{T}}^{v}$ will belong to $\mathfrak{F}_{o}$.

Remark 1 Alternatively, it is possible to verify directly that a recurrent sandpile configuration restricted to any set $\widetilde{\mathbb{T}}^{v} \backslash\{v\}$ is deterministic, and its height equals $\operatorname{deg}(w)-1$ at $w$. Hence if $v$ topples in a wave, all of $\widetilde{T}^{v}$ topples.

\subsection{Quenched lower bound on avalanche size}

Recall that given a supercritical Galton-Watson tree $\mathbb{T}$, we denoted by $v^{*}=v^{*}(\mathbb{T})$ the closest vertex to $o$ with the property that $v^{*}$ has at least two children with an infinite line of descent. Let $\mathbb{T}_{k}^{\prime}\left(\mathbb{T}_{\leq k}^{\prime}\right.$, etc.) denote the set of vertices in the $k$-th generation of $\mathbb{T}^{\prime}$ (in all generations up to generation $k$, etc.), respectively. That is, the smallest integer $k$ such that $\left|\mathbb{T}_{k+1}^{\prime}\right|>1$ is $\left|v^{*}\right|$.

The following theorem implies the quenched lower bound of Theorem 1 stated in the introduction.

Theorem 5 Underassumption (M- $\beta$ ) and $\mu=\sum_{k \geq 0} k p_{k}>1$, there exists $c_{0}=c_{0}(\mathbb{T})$ that is a.s. positive on the event when $\mathbb{T}$ survives, such that we have

$$
v_{\mathbb{T}}[S>t] \geq v_{\mathbb{T}}\left[\left|W^{1}(\eta)\right|>t\right] \geq v_{\mathbb{T}}\left[\left|W^{N-\left|v^{*}\right|}(\eta)\right|>t\right] \geq c_{0} t^{-1 / 2} .
$$

Proof The first inequality follows from (7) and the second one from Lemma 1 (iii). For the third inequality, assume the event that $\mathbb{T}$ survives. Let $y_{1}, \ldots, y_{\ell}$ be the children of $v^{*}$ with infinite line of descent, $\ell \geq 2$. Let $\mathscr{G}$ be the connected component of $o$ in $\mathbb{T} \backslash\left(\cup_{j=1}^{\ell} \mathbb{T}\left(y_{j}\right)\right)$, and note that $\mathscr{G}$ is a finite graph. We will use Wilson's algorithm to construct an event on which $v^{*}$ is in $\mathfrak{F}_{o}$ but $y_{1}$ is not. Let us use Wilson's algorithm 
with the walks $S^{(*)}, S^{(1)}, S^{(2)}$ started at $v^{*}, y_{1}, y_{2}$ respectively, in this order. Consider the event:

$$
U:=\left\{S^{(*)} \text { hits } o ; S^{(1)} \text { does not hit } v^{*} ; S^{(2)} \text { hits } v^{*}\right\}
$$

On this event $\mathfrak{F}_{o}$ will correspond to a wave with the property that $v^{*}$ topples, but at least one of its children, namely $y_{1}$, does not. Hence by Lemma 2 this wave is $W^{N-\left|v^{*}\right|}(\eta)$. Moreover, we have

$$
\mathfrak{F}_{o} \supset \mathscr{G} \cup \mathfrak{F}_{o}^{(2)}
$$

where $\mathfrak{F}_{o}^{(2)}$ is distributed as the $\mathrm{WSF}_{o}$ component of $y_{2}$ in $\mathbb{T}\left(y_{2}\right)$. To complete the proof we note that

$$
\begin{aligned}
v_{\mathbb{T}}\left[\left|W^{N-\left|v^{*}\right|}(\eta)\right|>t\right] & \geq P^{\mathbb{T}}\left[U,\left|\mathfrak{F}_{o}^{(2)}\right|>t\right]=P^{\mathbb{T}}[U] P^{\mathbb{T}\left(y_{2}\right)}\left[\left|\mathfrak{F}_{o}\right|>t\right] \\
& \geq c(\mathbb{T}) c\left(\mathbb{T}\left(y_{2}\right)\right) t^{-1 / 2}
\end{aligned}
$$

where the equality follows from the fact that, conditioned on $U, \mathfrak{F}_{o}^{(2)}$ is equal in law to $\mathfrak{F}_{o}$ on $\mathbb{T}\left(y_{2}\right)$. The final lower bound follows from the transience of the random walk on $\mathbb{T}\left(y_{1}\right)$ on the one hand, and on Theorem 4 on the other hand.

\subsection{Upper bound on avalanche size}

In this section we prove the following avalanche size bound.

Theorem 6 Assume that $1<\sum_{k \geq 0} k p_{k} \leq \infty$. There exists $C=C$ (p) and on the event $F$ an a.s. finite $N_{2}=N_{2}(\mathbb{T})$ such that for all $t \geq N_{2}$ we have

$$
P^{\mathbb{T}}[S>t] \leq C t^{-1 / 2}
$$

Recall that $N$ denotes the number of waves. This equals 1 plus the largest integer $k$, such that the first wave contains all vertices in the $k$-th generation of $\mathbb{T}$, see Lemma 1 (ii).

We use the notation $P_{v}^{\mathbb{T}}$ for the law of a simple random walk $\left\{S_{n}\right\}_{n \geq 0}$ on $\mathbb{T}$ with $S_{0}=v$. We denote the hitting time of a set $A$ by $\xi_{A}:=\inf \left\{n \geq 0: S_{n} \in A\right\}$.

Lemma 8 We have

$$
\nu_{\mathbb{T}}[N \geq k+1] \leq G^{\mathbb{T}}(o, o) \prod_{e: e^{+} \in \mathbb{T}_{k}^{\prime}} \frac{1}{1+\mathscr{C}\left(e^{+}\right)}, \quad k \geq 0
$$

where the empty product for $k=0$ is interpreted as 1 . 
Proof We can bound from above the probability that the first wave contains $\mathbb{T}_{\leq k}$ by $G^{\mathbb{T}}(o, o)$ times the probability that a typical wave contains it. Thus by Lemma 3

$$
v_{\mathbb{T}}[N \geq k+1] \leq G^{\mathbb{T}}(o, o) P^{\mathbb{T}}\left[\mathfrak{F}_{o} \supset \mathbb{T}_{\leq k}\right]=G^{\mathbb{T}}(o, o) P^{\mathbb{T}}\left[\mathfrak{F}_{o} \supset \mathbb{T}_{k}^{\prime}\right]
$$

In the last step, we used that $\mathbb{T}_{\leq k} \subset \mathfrak{F}_{o}$ if and only if $\mathbb{T}_{k}^{\prime} \subset \mathfrak{F}_{o}$. This is implied by Lemma 7 , since if $\mathfrak{F}_{o}$ misses a vertex $w \in \mathbb{T}_{\leq k}$, it will also necessarily miss an ancestor of $w$ lying in $\mathbb{T}_{\leq k}^{\prime}$, and hence will also miss a vertex of $\mathbb{T}_{k}^{\prime}$. Using Wilson's algorithm and Lemma 4 with walks started at vertices in $\mathbb{T}_{k}^{\prime}$, we get that the probability in the right hand side is at most

$$
\prod_{e: e^{+} \in \mathbb{T}_{k}^{\prime}} P_{e^{+}}^{\mathbb{T}}\left(\xi_{e^{-}}<\infty\right)=\prod_{e: e^{+} \in \mathbb{T}_{k}^{\prime}} \frac{1}{1+\mathscr{C}\left(e^{+}\right)}
$$

We denote by $\mathbf{p}^{\prime}=\left\{p_{k}^{\prime}\right\}_{k \geq 0}$ the offspring distribution of $\mathbb{T}^{\prime}$.

Lemma 9 Assume that $1<\sum_{k \geq 0} k p_{k} \leq \infty$.

(i) We can find a constant $C_{2}=C_{2}(\mathbf{p})$, and on the event $F$ an a.s. finite $K_{1}=$ $K_{1}\left(\mathbb{T}^{\prime}\right) \geq N_{1}\left(\mathbb{T}^{\prime}\right)$ such that for all $k \geq K_{1}$ we have

$$
\max \left\{N_{1}(\mathbb{T}(w)): w \in \mathbb{T}_{k}^{\prime}\right\} \leq C_{2}\left|\mathbb{T}_{k}^{\prime}\right|
$$

Moreover, we have $\mathbf{P}\left[K_{1} \geq k \mid F\right] \leq C \exp \left(-\delta_{0}^{\prime} k\right)$, where $\delta_{0}^{\prime}=\delta_{0}\left(\mathbf{p}^{\prime}\right)$ is the isoperimetric expansion constant of $\mathbf{p}^{\prime}$.

(ii) We can also find $C_{3}=C_{3}(\mathbf{p})$ and $c_{2}=c_{2}(\mathbf{p})>0$ such that for all $k \geq N_{1}\left(\mathbb{T}^{\prime}\right)$ we have

$$
(k+1)^{1 / 2}\left|\mathbb{T}_{k}^{\prime}\right|\left(\sum_{w \in \mathbb{T}_{k}^{\prime}} \overline{\mathscr{C}}(w)\right) \prod_{v \in \mathbb{T}_{k}^{\prime}} \frac{1}{1+\mathscr{C}(v)} \leq C_{3} \exp \left(-c_{2} k\right) .
$$

Proof (i) Conditioned on $\mathbb{T}_{\leq k}^{\prime}$, the trees $\left\{\mathbb{T}(w): w \in \mathbb{T}_{k}^{\prime}\right\}$ are independent, and the variables $N_{1}(\mathbb{T}(w))$ have an exponential tail, due to $(3)$. Hence we have

$$
\begin{aligned}
& \mathbf{P}\left[\max \left\{N_{1}(\mathbb{T}(w)): w \in \mathbb{T}_{k}^{\prime}\right\}>C_{2}\left|\mathbb{T}_{k}^{\prime}\right|\right] \\
& =\mathbf{E}\left[\mathbf{P}\left[\max \left\{N_{1}(\mathbb{T}(w)): w \in \mathbb{T}_{k}^{\prime}\right\}>C_{2}\left|\mathbb{T}_{k}^{\prime}\right| \mid \mathbb{T}_{\leq k}\right]\right] \\
& \leq \mathbf{E}\left[\sum_{w \in \mathbb{T}_{k}^{\prime}} \mathbf{P}\left[N_{1}(\mathbb{T}(w))>C_{2}\left|\mathbb{T}_{k}^{\prime}\right| \mid \mathbb{T}_{\leq k}^{\prime}\right]\right] \\
& \leq \mathbf{E}\left[\left|\mathbb{T}_{k}^{\prime}\right| C \exp \left(-c C_{2}\left|\mathbb{T}_{k}^{\prime}\right|\right)\right] .
\end{aligned}
$$


If $C_{2}>2 / c$, then the right hand side is at most

$$
C \mathbf{E}\left[\exp \left(-\left|\mathbb{T}_{k}^{\prime}\right|\right)\right]
$$

If $k \geq N_{1}\left(\mathbb{T}^{\prime}\right)$, then

$$
\left|\mathbb{T}_{k}^{\prime}\right| \geq \delta_{0}^{\prime}\left|\mathbb{T}_{<k}^{\prime}\right| \geq \delta_{0}^{\prime} k
$$

and hence (19) is summable in $k \geq 1$. Therefore, statement (i) follows from the Borel-Cantelli Lemma.

(ii) Let us write the sum over $w$, together with the product over $v$ in the form:

$$
\sum_{w \in \mathbb{T}_{k}^{\prime}} \frac{\overline{\mathscr{C}}(w)}{1+\mathscr{C}(w)} \prod_{v \in \mathbb{T}_{k}^{\prime}: v \neq w} \frac{1}{1+\mathscr{C}(v)} \leq \sum_{w \in \mathbb{T}_{k}^{\prime}} \prod_{v \in \mathbb{T}_{k}^{\prime}: v \neq w} \frac{1}{1+\mathscr{C}(v)}
$$

Assume $k \geq N_{1}\left(\mathbb{T}^{\prime}\right)$. Then Proposition 1 can be applied with $A=\mathbb{T}_{<k}^{\prime}$ (since $|A| \geq k \geq N_{1}\left(\mathbb{T}^{\prime}\right)$ ), and this gives that, for $\delta_{1}^{\prime}:=\delta_{1}\left(\mathbf{p}^{\prime}\right)$, we have at least for a proportion $\delta_{1}^{\prime}$ of the $\delta_{1}^{\prime}$-good vertices $v$ that

$$
\mathscr{C}(v)>\frac{\mathscr{C}(v)}{1+\mathscr{C}(v)} \geq \delta_{1}^{\prime}
$$

so for these $v$ we have $1 /(1+\mathscr{C}(v))<\left(1+\delta_{1}^{\prime}\right)^{-1}$. Therefore

$$
\prod_{v \in \mathbb{T}_{k}^{\prime}: v \neq w} \frac{1}{1+\mathscr{C}(v)} \leq\left(1+\delta_{1}^{\prime}\right)^{-\delta_{1}^{\prime}\left|\mathbb{T}_{k}^{\prime}\right|+1} \leq\left(1+\delta_{1}^{\prime}\right) \exp \left(-\left(\delta_{1}^{\prime}\right)^{2}\left|\mathbb{T}_{k}^{\prime}\right|\right)
$$

Thus the left hand side expression in (18) is bounded above by

$$
(k+1)^{1 / 2}\left|\mathbb{T}_{k}^{\prime}\right|\left(1+\delta_{1}^{\prime}\right) \exp \left(-\left(\delta_{1}^{\prime}\right)^{2}\left|\mathbb{T}_{k}^{\prime}\right|\right) \text {. }
$$

Since $\left|\mathbb{T}_{k}^{\prime}\right| \geq \delta_{0}^{\prime} k$, the statement follows.

In what follows, we write

$$
\mathscr{T}=\mathscr{T}(k):=\mathbb{T}_{<k}^{\prime} \cup\left(\cup_{v \in \mathbb{T}_{<k}^{\prime}} \widetilde{\mathbb{T}}^{v}\right)
$$

Lemma 10 Assume that $1<\sum_{k \geq 0} k p_{k} \leq \infty$. There exists an a.s. finite $K_{2}=$ $K_{2}\left(\mathbb{T}^{\prime}\right) \geq K_{1}$ such that for all $k \geq K_{2}\left(\mathbb{T}^{\prime}\right)$ we have

$$
|\mathscr{T}| \leq(1 / \alpha)\left|\mathbb{T}_{<k}^{\prime}\right| \leq\left(\alpha \delta_{0}\right)^{-1}\left|\mathbb{T}_{k}^{\prime}\right|
$$

Moreover,

$$
\mathbf{P}\left[K_{2} \geq k\right] \leq C \exp (-c k) .
$$


Proof Note that the size of $\widetilde{T}^{o}$ has an exponential tail; see for example [11, Theorem 13.1]. Thus there exists $\lambda_{0}=\lambda_{0}(\mathbf{p})>0$ such that

$$
\mathbf{E}\left[\exp \left(\lambda_{0}\left|\widetilde{\mathbb{T}}^{o}\right|\right)\right]=: C\left(\lambda_{0}\right)<\infty
$$

Let $0<b \leq 1 / 2$ be a number that we fix with the property that

$$
C\left(\lambda_{0}\right)^{b} \leq e^{\lambda_{0} / 4}
$$

Conditionally on $\mathbb{T}_{\leq k}^{\prime}$, the trees $\left\{\widetilde{\mathbb{T}}^{v}: v \in \mathbb{T}_{<k}^{\prime}\right\}$ are i.i.d. with the distribution of $\widetilde{\mathbb{T}}^{o}$. Therefore, for $\alpha:=b /(1+b) \leq 1 / 3$, using (23) and (24), we have that

$$
\begin{aligned}
\mathbf{P}\left[|\mathscr{T}|>(1 / \alpha)\left|\mathbb{T}_{<k}^{\prime}\right|\right] & =\sum_{A} \mathbf{P}\left[\mathbb{T}_{<k}^{\prime}=A\right] \mathbf{P}\left[|\mathscr{T}|>(1 / \alpha)|A| \mid \mathbb{T}_{<k}^{\prime}=A\right] \\
& =\sum_{A} \mathbf{P}\left[\mathbb{T}_{<k}^{\prime}=A\right] \mathbf{P}\left[\sum_{v \in A}\left|\tilde{\mathbb{T}}^{v}\right|>(1 / b)|A|\right] \\
& \leq \sum_{A} \mathbf{P}\left[\mathbb{T}_{<k}^{\prime}=A\right] e^{-\lambda_{0}(1 / b)|A|} C\left(\lambda_{0}\right)^{|A|} \\
& \leq \sum_{A} \mathbf{P}\left[\mathbb{T}_{<k}^{\prime}=A\right] e^{-(3 / 4)\left(\lambda_{0} / b\right)|A|} \leq e^{-(3 / 4) k \lambda_{0} / b} .
\end{aligned}
$$

Thus the claim follows from the Borel-Cantelli Lemma.

Proof of Theorem 6 In the course of the proof we are going to choose $\bar{K}=\bar{K}(t) \geq K_{2}$ (recall $K_{2}$ defined in Lemma 10). We can then write:

$$
P^{\mathbb{T}}[S>t] \leq P^{\mathbb{T}}[N \geq \bar{K}+1]+P^{\mathbb{T}}\left[1 \leq N \leq K_{2}, S>t\right]+\sum_{K_{2} \leq k<\bar{K}} P^{\mathbb{T}}[N=k+1, S>t]
$$

The first term in the right hand side of (25) can be bounded using Lemma 8:

$$
P^{\mathbb{T}}[N \geq \bar{K}+1] \leq G^{\mathbb{T}}(o, o) \prod_{e: e^{+} \in \mathbb{T}_{\bar{K}}^{\prime}} \frac{1}{1+\mathscr{C}\left(e^{+}\right)}
$$

Since $\bar{K} \geq K_{1} \geq N_{1}\left(\mathbb{T}^{\prime}\right)$, we can apply Proposition 1 to $A=\mathbb{T}_{<k}^{\prime}$, and hence

$$
P^{\mathbb{T}}[N \geq \bar{K}+1] \leq G^{\mathbb{T}}(o, o)\left(1+\delta_{1}^{\prime}\right)^{-\delta_{1}^{\prime}\left|\mathbb{T}_{\bar{K}}^{\prime}\right|} \leq G^{\mathbb{T}}(o, o) \exp \left(-\left(\delta_{1}^{\prime}\right)^{2}\left|\mathbb{T}_{\bar{K}}^{\prime}\right|\right)
$$

Let us choose

$$
\bar{K}=\min \left\{k \geq 0:\left|\mathbb{T}_{k}^{\prime}\right| \geq C_{3} \log t\right\}
$$


where $C_{3}=C_{3}\left(\mathbf{p}^{\prime}\right):=\left[2\left(\delta_{1}^{\prime}\right)^{2}\right]^{-1}$. With this choice, we have

$$
P^{\mathbb{T}}[N \geq \bar{K}+1] \leq G^{\mathbb{T}}(o, o) t^{-1 / 2} .
$$

We turn to the second term in the right hand side of (25). Since $S(\eta)=\left|W^{1}(\eta)\right|+$ $\cdots+\left|W^{N}(\eta)\right|$, where $W^{1}(\eta) \supset \cdots \supset W^{N}(\eta)$, see Lemma 1(iii), we can write

$$
P^{\mathbb{T}}\left[1 \leq N \leq K_{2}, S>t\right] \leq P^{\mathbb{T}}\left[\left|W^{1}(\eta)\right|>t / K_{2}\right] \leq G^{\mathbb{T}}(o, o) P^{\mathbb{T}}\left[\left|\mathfrak{F}_{o}\right|>t / K_{2}\right],
$$

where we used Lemma 3 in the last step. An application of Theorem 3 gives:

$$
P^{\mathbb{T}}\left[1 \leq N \leq K_{2}, S>t\right] \leq C_{1} G^{\mathbb{T}}(o, o) N_{1}^{1 / 2} \overline{\mathscr{C}}(o) K_{2}^{1 / 2} t^{-1 / 2}
$$

Finally, we bound the third term in the right hand side of (25). Let $K_{2} \leq k<\bar{K}$. Using again (7), Lemmas 1 and 3, we can write

$$
\begin{aligned}
P^{\mathbb{T}}[N=k+1, S>t] & \leq P^{\mathbb{T}}\left[N=k+1,\left|W^{1}(\eta)\right|>\frac{t}{k+1}\right] \\
& \leq P^{\mathbb{T}}\left[N \geq k+1,\left|W^{1}(\eta)\right|>\frac{t}{k+1}\right] \\
& =P^{\mathbb{T}}\left[W^{1}(\eta) \supset \mathbb{T}_{k}^{\prime},\left|W^{1}(\eta)\right|>\frac{t}{k+1}\right] \\
& \leq G^{\mathbb{T}}(o, o) P^{\mathbb{T}}\left[\mathfrak{F}_{o} \supset \mathbb{T}_{k}^{\prime},\left|\mathfrak{F}_{o}\right|>\frac{t}{k+1}\right] \\
& =G^{\mathbb{T}}(o, o) P^{\mathbb{T}}\left[\mathfrak{F}_{o} \supset \mathbb{T}_{k}^{\prime}\right] P^{\mathbb{T}}\left[\left|\mathfrak{F}_{o}\right|>\frac{t}{k+1} \mid \mathfrak{F}_{o} \supset \mathbb{T}_{k}^{\prime}\right] .
\end{aligned}
$$

An application of Lemma 8 yields that

$$
P^{\mathbb{T}}\left[\mathfrak{F}_{o} \supset \mathbb{T}_{k}^{\prime}\right] \leq \prod_{v \in \mathbb{T}_{k}^{\prime}} \frac{1}{1+\mathscr{C}(v)}
$$

We proceed to bound the conditional probability in the right hand side of (28). For any $w \in \mathbb{T}_{k}^{\prime}$, let us write $\mathfrak{F}_{o, w}=\mathfrak{F}_{o} \cap \mathbb{T}(w)$. This way, conditionally on $\mathfrak{F}_{o} \supset \mathbb{T}_{k}^{\prime}$, we have

$$
\mathfrak{F}_{o}=\mathscr{T} \cup\left(\bigcup_{w \in \mathbb{T}_{k}^{\prime}} \mathfrak{F}_{o, w}\right)
$$

where $\mathscr{T}$ was defined in (21), and where the conditional distribution of $\mathfrak{F}_{o, w}$ equals that of $\mathfrak{F}_{o}(\mathbb{T}(w))$. Then, using the restriction $k \geq K_{2}$, we have

$$
P^{\mathbb{T}}\left[\left|\mathfrak{F}_{o}\right|>\frac{t}{k+1} \mid \mathfrak{F}_{o} \supset \mathbb{T}_{k}^{\prime}\right] \leq P^{\mathbb{T}}\left[\sum_{w \in \mathbb{T}_{k}^{\prime}}\left|\mathfrak{F}_{o, w}\right|>\frac{t}{k+1}-|\mathscr{T}| \mid \mathfrak{F}_{o} \supset \mathbb{T}_{k}^{\prime}\right]
$$




$$
\begin{aligned}
& \leq P^{\mathbb{T}}\left[\sum_{w \in \mathbb{T}_{k}^{\prime}}\left|\mathfrak{F}_{o, w}\right|>\frac{t}{k+1}-\left(\alpha \delta_{0}\right)^{-1}\left|\mathbb{T}_{k}^{\prime}\right| \mid \mathfrak{F}_{o} \supset \mathbb{T}_{k}^{\prime}\right] \\
& \leq \sum_{w \in \mathbb{T}_{k}^{\prime}} P^{\mathbb{T}(w)}\left[\left|\mathfrak{F}_{o}(\mathbb{T}(w))\right|>\frac{t}{(k+1)\left|\mathbb{T}_{k}^{\prime}\right|}-\left(\alpha \delta_{0}\right)^{-1}\right] \\
& \leq \sum_{w \in \mathbb{T}_{k}^{\prime}} P^{\mathbb{T}(w)}\left[\left|\mathfrak{F}_{o}(\mathbb{T}(w))\right|>\frac{t}{2(k+1)\left|\mathbb{T}_{k}^{\prime}\right|}\right] .
\end{aligned}
$$

In the second inequality we use (22) and in the last step we use that on the one hand $k<\bar{K}$ implies $\left|\mathbb{T}_{k}^{\prime}\right|<C_{3} \log t$ and on the other hand $k \leq\left|\mathbb{T}_{<k}^{\prime}\right| \leq\left(\delta_{0}^{\prime}\right)^{-1}\left|\mathbb{T}_{k}^{\prime}\right| \leq$ $\left(\delta_{0}^{\prime}\right)^{-1} C_{3} \log t$ (cf. (20)) and hence the inequality follows for $t \geq t_{1}=t_{1}$ (p).

Applying Theorem 3 to the probability in the right hand side of (30) yields the upper bound

$$
C^{\prime} t^{-1 / 2}(k+1)^{1 / 2}\left|\mathbb{T}_{k}^{\prime}\right|^{1 / 2} \sum_{w \in \mathbb{T}_{k}^{\prime}} \overline{\mathscr{C}}(w) N_{1}^{1 / 2}(\mathbb{T}(w))
$$

Due to $k \geq K_{2} \geq K_{1}$, and Lemma 9(i), this expression is at most

$$
C^{\prime \prime} t^{-1 / 2}(k+1)^{1 / 2}\left|\mathbb{T}_{k}^{\prime}\right| \sum_{w \in \mathbb{T}_{k}^{\prime}} \overline{\mathscr{C}}(w)
$$

Substituting (29) and (31) into the right hand side of (28) and using Lemma 9(ii) yields

$$
\sum_{K_{2} \leq k<\bar{K}} P^{\mathbb{T}}[N=k+1, S>t] \leq C t^{-1 / 2} G^{\mathbb{T}}(o, o) \sum_{k \geq K_{2}} \exp (-c k) \leq C G^{\mathbb{T}}(o, o) t^{-1 / 2} .
$$

The inequalities (26), (27) and (32) substituted into (25) complete the proof of the theorem.

\section{Annealed bounds}

Finally, we prove annealed bounds.

Theorem 7 (i) Under Assumption $(\mathrm{M}-\beta)$, there exists $c=c(\mathbf{p})>0$ such that

$$
\mathbf{E}\left[v_{\mathbb{T}}[S>t] \mid \mathbb{T} \text { survives }\right] \geq \mathbf{E}\left[v_{\mathbb{T}}[|\operatorname{Av}(\eta)|>t] \mid \mathbb{T} \text { survives }\right] \geq c t^{-1 / 2}
$$

(ii) Assume that $1<\sum_{k \geq 0} k p_{k} \leq \infty$. There exists $C=C$ (p) such that

$$
\mathbf{E}\left[\nu_{\mathbb{T}}[|\operatorname{Av}(\eta)|>t] \mid \mathbb{T} \text { survives }\right] \leq \mathbf{E}\left[v_{\mathbb{T}}[S>t] \mid \mathbb{T} \text { survives }\right] \leq C t^{-1 / 2}
$$


Proof Part (i) follows immediately after taking expectations in (17) of Theorem 5.

For part (ii), we take expectations in the right hand sides of (26), (27) and (32). We detail the bound on the expectation of (27), the other two are similar and simpler. Recall the notation $\mathscr{C}(o)$ in $(7)$, and $\overline{\mathscr{C}}(o)$ in (9). We similarly denote by $\mathscr{R}(o)$ the effective resistance in $\mathbb{T}$ from $o$ to infinity. We have $G^{\mathbb{T}}(o, o)=\mathscr{R}(o)$. Therefore, $G^{\mathbb{T}}(o, o) \overline{\mathscr{C}}(o)=\max \{1, \mathscr{R}(o)\}$, and we need to bound the expectation of

$$
\max \{1, \mathscr{R}(o)\} N_{1}^{1 / 2} K_{2}^{1 / 2} \text {. }
$$

Here $N_{1}$ has an exponential tail, due to (3), and $K_{2}$ has an exponential tail due to Lemma 10 . We now show that $\mathscr{R}(o)$ also has an exponential tail, which immediately implies that the expectation is finite.

First observe that $\mathscr{R}(o)$ is also the effective resistance in $\mathbb{T}^{\prime}$ from $o$ to infinity, hence we may restrict to $\mathbb{T}^{\prime}$. Recall that $\left\{p_{k}^{\prime}\right\}_{k \geq 0}$ denotes the offspring distribution of $\mathbb{T}^{\prime}$. In the case $p_{1}^{\prime}=0$, there is at least binary branching, and hence $\mathscr{R}(o) \leq 1$. Henceforth we assume $0<p_{1}^{\prime}<1$. Let $v_{\emptyset}$ be the first descendant of $o$ in $\mathbb{T}^{\prime}$, where the tree branches, that is, there are single offspring until $v_{\emptyset}$, but $v_{\emptyset}$ has at least two offspring. Consider only the first two offspring of $v_{\emptyset}$. Let $v_{1}$ and $v_{2}$ be the first descendants of $v_{\emptyset}$ where branching occurs, that is, each individual on the path between $v_{\emptyset}$ and $v_{i}$ has a single offspring, but $v_{i}$ has at least two offspring $(i=1,2)$. Analogously, we define $v_{\varepsilon_{1}, \ldots, \varepsilon_{k}}$ for each $\left(\varepsilon_{1}, \ldots, \varepsilon_{k}\right) \in\{1,2\}^{k}, k \geq 0$.

Let $R_{\emptyset}$ be the resistance between $o$ and $v_{\emptyset}$ (this is the same as the generation difference, since each edge has resistance 1), let $R_{v_{i}}$ be the resistance between $v_{\emptyset}$ and $v_{i}$ (for $i=1,2$ ) and more generally let $R_{\varepsilon_{1}, \ldots, \varepsilon_{k}}$ be the resistance between $v_{\varepsilon_{1}, \ldots, \varepsilon_{k-1}}$ and $v_{\varepsilon_{1}, \ldots, \varepsilon_{k}}$ for $k \geq 1$. These random variables are independent, and apart from $R_{\emptyset}$, they are identically distributed with distribution $\mathbf{P}\left[R_{\varepsilon_{1}, \ldots, \varepsilon_{k}}=r\right]=\left(p_{1}^{\prime}\right)^{r-1}\left(1-p_{1}^{\prime}\right)$, $r \geq 1$. The variable $R_{\emptyset}$ has distribution: $\mathbf{P}\left[R_{\emptyset}=r\right]=\left(p_{1}^{\prime}\right)^{r}\left(1-p_{1}^{\prime}\right), r \geq 0$.

For any $0<t<-\log \left(p_{1}^{\prime}\right)$ the resistance variables all satisfy the bound

$$
\mathbf{E}\left[\exp \left(t R_{\varepsilon_{1}, \ldots, \varepsilon_{k}}\right)\right] \leq \varphi(t):=\frac{1-p_{1}^{\prime}}{p_{1}^{\prime}} \frac{p_{1}^{\prime} e^{t}}{1-p_{1}^{\prime} e^{t}}=\frac{\left(1-p_{1}^{\prime}\right) e^{t}}{1-p_{1}^{\prime} e^{t}}
$$

We fix $t_{0}=-\frac{1}{2} \log \left(p_{1}^{\prime}\right)>0$, so that for all $0<t \leq t_{0}$ the right hand side is bounded above by $\left(1+\sqrt{p_{1}}\right) / \sqrt{p_{1}}=\sqrt{C_{2}^{\prime}}<\infty$.

By the series and parallel laws, the resistance between $o$ and $\left\{v_{1}, v_{2}\right\}$ is

$$
R_{\emptyset}+\frac{1}{\frac{1}{R_{1}}+\frac{1}{R_{2}}}
$$

By the inequality between the harmonic mean and arithmetic mean, (33) can be bounded above by

$$
R_{\emptyset}+\frac{1}{2} \frac{1}{\frac{\frac{1}{R_{1}}+\frac{1}{R_{2}}}{2}} \leq R_{\emptyset}+\frac{1}{2} \frac{R_{1}+R_{2}}{2}=R_{\emptyset}+\frac{R_{1}}{4}+\frac{R_{2}}{4} .
$$


Iterating this argument, we get for the effective resistance $\mathscr{R}(o)$ between $o$ and infinity,

$$
\mathscr{R}(o) \leq R_{\emptyset}+\frac{1}{4}\left(R_{1}+R_{2}\right)+\frac{1}{16}\left(R_{1,1}+R_{1,2}+R_{2,1}+R_{2,2}\right)+\cdots .
$$

Consequently, by Jensen's inequality, we have

$$
\begin{aligned}
\mathbf{E}[\exp (t \mathscr{R}(o))] & \leq \mathbf{E}\left[\exp \left(t R_{1}\right)\right] \mathbf{E}\left[\exp \left(\frac{t}{2} R_{1}\right)\right] \mathbf{E}\left[\exp \left(\frac{t}{4} R_{1,1}\right)\right] \cdots \\
& \leq \mathbf{E}\left[\exp \left(t R_{1}\right)\right] \mathbf{E}\left[\exp \left(t R_{1}\right)\right]^{1 / 2} \mathbf{E}\left[\exp \left(t R_{1,1}\right)\right]^{1 / 4} \ldots \\
& \leq \varphi(t)^{1+\frac{1}{2}+\frac{1}{4}+\cdots} \\
& =\varphi(t)^{2} \leq C_{2}^{\prime}, \quad 0<t \leq t_{0} .
\end{aligned}
$$

This yields the claimed exponential decay, and the proof is complete.

Acknowledgements The authors thank the following institutions for hospitality and support: MAP5 lab. at Université Paris Descartes, Bath University, Delft University as well as Institut Henri Poincaré (UMS 5208 CNRS-Sorbonne Université, endowed with LabEx CARMIN, ANR-10-LABX-59-01) - Centre Emile Borel, where part of this work was done during the trimester "Stochastic Dynamics Out of Equilibrium". Finally, the authors would like to thank the anonymous referee for suggestions that improved the manuscript significantly.

Open Access This article is distributed under the terms of the Creative Commons Attribution 4.0 International License (http://creativecommons.org/licenses/by/4.0/), which permits unrestricted use, distribution, and reproduction in any medium, provided you give appropriate credit to the original author(s) and the source, provide a link to the Creative Commons license, and indicate if changes were made.

\section{References}

1. Aldous, D.J., Lyons, R.: Processes on unimodular random networks. Electron. J. Probab. 12, 1454-1508 (2007)

2. Athreya, S.R., Járai, A.A.: Infinite volume limit for the stationary distribution of abelian sandpile models. Commun. Math. Phys. 249, 197-213 (2004)

3. Bak, P., Tang, C., Wiesenfeld, K.: Self-organized criticality. Phys. Rev. A 38, 364-374 (1988)

4. Beggs, J.M., Plenz, D.: Neuronal avalanches in neocortical circuits. J. Neurosci. 23, 11167-11177 (2003)

5. Bhupatiraju, S., Hanson, J., Járai, A.A.: Inequalities for critical exponents in $d$-dimensional sandpiles. Electron. J. Probab. 22(85) (2017). https://doi.org/10.1214/17-EJP111

6. Chen, D., Peres, Y.: Anchored expansion, percolation and speed. Ann. Prob. 32, 2978-2995 (2004)

7. Cori, R., Le Borgne, Y.: The sandpile model and Tutte polynomials. Adv. Appl. Math. 30(1-2), 44-52 (2003)

8. Dhar, D.: Self-organized critical state of sandpile automaton models. Phys. Rev. Lett. 64, 1613-1616 (1990)

9. Dhar, D.: Theoretical studies of self-organized criticality. Phys. A 369, 29-70 (2006)

10. Dhar, D., Majumdar, S.N.: Abelian sandpile model on the Bethe lattice. J. Phys. A: Math. Gen. 23, 4333-4350 (1990)

11. Harris, T.E.: The Theory of Branching Processes. Springer, Berlin (1963)

12. Holroyd, A.E., Levine, L., Mészáros, K., Peres, Y., Propp, J., Wilson, D.B.: Chip-firing and rotorrouting on directed graphs. In: Sidoravicius, V., Vares, M.E. (eds.) In and out of Equilibrium, vol. 2, pp. 361-364. Basel, Brikhäuser (2008) 
13. Hutchcroft, T.: Interlacements and the wired uniform spanning forest. Ann. Probab. 46(2), 1170-1200 (2018)

14. Hutchcroft, T.: Universality of high-dimensional spanning forests and sandpiles. Probab. Theory Relat. Fields (to appear)

15. Ivashkevich, E.V., Ktitarev, D.V., Priezzhev, V.B.: Waves of topplings in an abelian sandpile. Phys. A 209, 347-360 (1994)

16. Janowsky, S.A., Laberge, C.A.: Exact solutions for a mean-field Abelian sandpile. J. Phys. A: Math. Gen. 26(19), L973 (1993)

17. Járai, A.A.: Sandpile models. Probab. Surv. 15, 243-306 (2018)

18. Járai, A.A., Redig, F.: Infinite volume limit of the abelian sandpile model in dimensions $d \geq 3$. Probab. Theory Relat. Fields 141, 181-212 (2008)

19. Járai, A.A., Redig, F., Saada, E.: Approaching criticality via the zero dissipation limit in the abelian avalanche model. J. Stat. Phys. 159(6), 1369-1407 (2015)

20. Járai, A.A., Werning, N.: Minimal configurations and sandpile measures. J. Theor. Prob. 27(1), 153-167 (2014)

21. Kirchhoff, G.: Über die Auflösung der Gleichungen, auf welche man bei der Untersuchung der linearen Verteilung Galvanischer Ströme geführt wird. Ann. Phys. 148(12), 497-508 (1847)

22. Lyons, R., Morris, B., Schramm, O.: Ends in uniform spanning forest. Electron. J. Probab. 13(58), 1702-1725 (2008)

23. Lyons, R., Peres, Y.: Probability on Trees and Networks. Cambridge University Press, Cambridge (2016)

24. Maes, C., Redig, F., Saada, E.: The Abelian sandpile model on an infinite tree. Ann. Prob. 30(4), 2081-2107 (2002)

25. Majumdar, S.N., Dhar, D.: Equivalence between the Abelian sandpile model and the $q \rightarrow 0$ limit of the Potts model. Phys. A 185, 129-145 (1992)

26. Morris, B.: The components of the wired spanning forest are recurrent. Probab. Theory Relat. Fields 125, 259-265 (2003)

27. Redig, F.: Mathematical aspects of the abelian sandpile model. Les Houches lecture notes in Mathematical statistical physics., 657-729, Session XXXIII, Elsevier B. V., Amsterdam (2006)

28. Redig, F., Ruszel, W.M., Saada, E.: The abelian sandpile model on a random binary tree. J. Stat. Phys. 147(4), 653-677 (2012)

29. Redig, F., Ruszel, W.M., Saada, E.: Non-criticality of the Abelian sandpile model on a random tree and related models. J. Math. Phys. 59(6), 1-16 (2018)

30. Ruelle, P.: Logarithmic conformal invariance in the Abelian sandpile model. J. Phys. A: Math. Theor. 46, 494014 (2013)

31. Wilson, D.B.: Generating random spanning trees more quickly than the cover time. In: Proceedings of the Twenty-Eights Annual ACM Symposium on the Theory of Computing. pp. 296-303, ACM, New York (1996)

Publisher's Note Springer Nature remains neutral with regard to jurisdictional claims in published maps and institutional affiliations. 